\title{
L'analyse du contenu textuel en vue de la construction de thésaurus et de l'indexation assistées par ordinateur; applications possibles avec SATO
}

The Analysis of Textual Content With a View to the Construction of a Thesaurus and Computer-Based indexation: Applications Using SATO

Análisis del contenido textual para la elaboración de un
diccionario de sinónimos y repertorios de índices asistida por
computadora; Aplicaciones posibles con SATO

Suzanne Bertrand-Gastaldy et Gracia Pagola

Volume 38, numéro 2, avril-juin 1992

Analyse et gestion de l'information textuelle

URI : https://id.erudit.org/iderudit/1028612ar

DOI : https://doi.org/10.7202/1028612ar

\section{Aller au sommaire du numéro}

\section{Éditeur(s)}

Association pour l'avancement des sciences et des techniques de la documentation (ASTED)

\section{ISSN}

0315-2340 (imprimé)

2291-8949 (numérique)

Découvrir la revue

\section{Citer cet article}

Bertrand-Gastaldy, S. \& Pagola, G. (1992). L'analyse du contenu textuel en vue de la construction de thésaurus et de l'indexation assistées par ordinateur; applications possibles avec SATO. Documentation et bibliothèques, 38(2), 75-89. https://doi.org/10.7202/1028612ar
Résumé de l'article

L'intervention de l'ordinateur, longtemps réservée aux tâches mécaniques effectuées en aval de l'analyse des documents et de la constitution des thésaurus, se déplace en amont vers l'analyse elle-même. Des logiciels existent désormais qui assistent l'exploration des textes. On montre comment SATO (système d'analyse de textes par ordinateur), utilisé par des chercheurs de plusieurs disciplines, peut faciliter les tâches de contrôle et de structuration du vocabulaire ainsi que l'indexation. On présente ses caractéristiques importantes : possibilité d'ajouter des propriétés aux mots et aux segments textuels, génération de lexiques, analyses lexico-statistiques diverses, définition de sous-ensembles de textes et de lexiques. On examine ensuite l'aide apportée dans l'élaboration de thésaurus : extraction d'unités lexicales simples et complexes, pondération pour faciliter le choix, regroupements divers, repérage en contexte de formes équivalentes, de synonymes, de termes génériques et spécifiques, de termes associés. L’indexation assistée par ordinateur est également illustrée, avec des perspectives d'analyse « sur mesure ", de même que plusieurs stratégies d'interrogation. On conclut sur la nécessité de résoudre les questions théoriques auxquelles l'analyse du contenu confronte désormais les spécialistes de l'information.
Tous droits réservés $@$ Association pour l'avancement des sciences et des techniques de la documentation (ASTED), 1992
Ce document est protégé par la loi sur le droit d'auteur. L’utilisation des services d'Érudit (y compris la reproduction) est assujettie à sa politique d'utilisation que vous pouvez consulter en ligne.

https://apropos.erudit.org/fr/usagers/politique-dutilisation/ 


\title{
L'analyse du contenu textuel en vue de la construction de thésaurus et de l'indexation assistées par ordinateur ; applications possibles avec SATO
}

\author{
Suzanne Bertrand-Gastaldy* \\ Gracia Pagola**
}

\begin{abstract}
L'intervention de l'ordinateur, longtemps réservée aux tâches mécaniques effectuées en aval de l'analyse des documents et de la constitution des thésaurus, se déplace en amont vers l'analyse elle-même. Des logiciels existent désormais qui assistent l'exploration des textes. On montre comment SATO (système d'analyse de textes par ordinateur), utilisé par des chercheurs de plusieurs disciplines, peut faciliter les tâches de contrôle et de structuration du vocabulaire ainsi que l'indexation. On présente ses caractéristiques importantes: possibilité d'ajouter des propriétés aux mots et aux segments textuels, génération de lexiques, analyses lexico-statistiques diverses, définition de sous-ensembles de textes et de lexiques. On examine ensuite l'aide apportée dans l'élaboration de thésaurus: extraction d'unités lexicales simples et complexes, pondération pour faciliter le choix, regroupements divers, repérage en contexte de formes équivalentes, de synonymes, de termes génériques et spécifiques, de termes associés. L'indexation assistée par ordinateur est également illustrée, avec des perspectives d'analyse "sur mesure", de même que plusieurs stratégies d'interrogation. On conclut sur la nécessité de résoudre les questions théoriques auxquelles l'analyse du contenu confronte désormais les spécialistes de l'information.
\end{abstract}

\section{The Analysis of Textual Content With a View to the Construction of a Thesaurus and Computer-Based Indexation: Applications Using SATO}

The use of the computer, formerly reserved for the mechanical manipulation prior to subject analysis and thesaurus construction, is now shifting toward the analysis itself. Current software is now capable of assisting subject analysis. The authors demonstrate how SATO (Système d'analyse de textes par ordinateur), used by researchers in several fields, can help in the tasks of vocabulary control and structure as well as indexation. Its principle characteristics are outlined: the possibility of adding the characteristics of words and parts of the text, the creation of glossaries, various glossary-based statistical analyses, the definition of sub-sets of texts and glossaries. They then examine the aid used in thesaurus construction: the extraction of simple and complex glossary units, weighed to assist in the choice, assorted groupings, contextually equivalent forms retrieved, synonyms, generic and specific terms, associated terms. Computerassisted indexation is also outlined along with "tailor-made" subject analysis, and search strategies. The conclusion calls for the necessity to resolve the theoretical questions of subject analysis currently facing information specialists.

\begin{abstract}
Análisis del contenido textual para la elaboración de un diccionario de sinónimos y repertorios de índices asistida por computadora; Aplicaciones posibles con SATO
\end{abstract}

La intervención de la computadora, durante mucho tiempo reservada a tareas mecánicas efectuadas para el análisis de documentos y la elaboración de un diccionario de sinónimos, se dirige hacia el análisis propiamente dicho. Actualmente hay programas que asisten en la exploración de textos. Se muestra de qué manera SATO (sistema de análisis de textos por computadora), utilizado por los investigadores de numerosas disciplinas, puede facilitar las tareas de control y estructuración del vocabulario e índices. Se presentan las características importantes: posibilidad de agregar propiedades a las palabras $y$ a los segmentos textuales, producción de léxicos, análisis léxicoestadísticos diversos, definición de subconjuntos de textos y léxicos. A continuación se examina la ayuda que aporta en la elaboración del diccionario de sinónimos: extracción de unidades léxicas simples y complejas, ponderación para facilitar la selección, reagrupamientos diversos, localización en contexto de formas equivalentes, sinónimos, términos genéricos y específicos y términos asociados. Se ilustra igualmente la indexación asistida por computadora, con perspectiva de análisis "a medida", y numerosas estrategias de interrogación. Se concluye con la necesidad de resolver los aspectos teóricos sobre el análisis del contenido a los cuales habrán de enfrentarse a partir de ahora los especialistas de la información.
L'intervention de l'ordinateur a été longtemps réservée aux tâches mécaniques, notamment à celles qui sont effectuées en a aval de l'analyse des documents et de la constitution des thésaurus, mais avec la disponibilité des textes sur ordinateur cette intervention se déplace en amont, vers l'analyse elle-même, vers l'exploration du contenu des textes.

La construction de vocabulaires contrôlés et l'indexation, loin de disparaître avec la multiplication des bases de données en plein texte, comme certains l'avaient prédit à la fin des années 1970, connaissent au contraire un regain d'intérêt. Information Processing \& Management a consacré, en 1990, tout un numéro à l'analyse textuelle et une livraison entière de International Classification (no3-4, 1990) a été réservée aux logiciels de gestion de thésaurus. La lecture de publications récentes confirme que l'intérêt gagne même les cercles non bibliothéconomiques: dans le numéro d'avril 1991 de Byte sur le bureau sans papier ( « Paperless

\footnotetext{
* Professeure agrégée à l'École de bibliothéconomie et des sciences de l'information de l'Université de Montréal et chercheure au Centre d'ATO (Analyse de textes par ordinateur) de I'UQAM (Université du Québec à Montréal).

* Agente de recherche et chargée de cours à l'École de bibliothéconomie et des sciences de l'information de l'Université de Montréal.
} 
Office "), Locke entreprend de convaincre les gestionnaires qui ont opté pour un système de gestion électronique des documents (GED en français et DIP "Document Image Processing " en anglais) de la nécessité de l'indexation et du contrôle de vocabulaire pour accéder au contenu des textes. II souligne la nature complexe de la tâche "[...] indexing is not a low-level task, and it becomes more complex as larger volumes of text are involved "1. II ajoute: "In fact, the subject analysis that librarians perform to create these categories and relationships is strongly akin to what the Al literature calls knowledge engineering".

Parce que le contenu des bases de données textuelles ne peut pas être exploité de façon satisfaisante pour les utilisateurs de plus en plus nombreux et diversifiés avec les méthodes traditionnelles de mots clés ou de chaînes de caractères des systèmes bibliographiques, les spécialistes en sciences de I'information n'ont d'autre choix que de collaborer avec les autres spécialistes de la langue et des textes. Leurs travaux trouvent naturellement une place dans les industries de la langue, comme on peut le constater d'après le programme des congrès sur ce thème (Colloque "Les industries de la langue: Perspectives des années 1990 " en novembre 1990 à Montréal). II ne s'agit pas de leur part d'une démarche opportuniste, mais bien de la reconnaissance de la nature linguistique du matériau à traiter (BertrandGastaldy, 1990a). D'ailleurs la collaboration ne s'effectue pas à sens unique. Les thésaurus ou des outils approchants deviennent nécessaires aux linguistes pour le traitement automatique des langues:

In a rather rough statement one could say that is now the linguists, who on the one hand, need thesauri, or thesaurus-like conceptual structures, to solve their problem of meaning, i.e. the problem of language understanding, whereas the IR [information retrieval] systems designers, in turn, finally came to know that basic LE [linguistic engineering] is required in their systems to come up with more efficient, intelligent, machine-aided IR systems ${ }^{2}$.

De plus en plus de parallèles sont établis d'une part entre les thésaurus et les méthodes d'indexation avec grilles et, d'autre part, certains modes de représentation des connaissances en intelligence artificielle, comme les réseaux sémantiques et les "frames".

Bien qu'un faible pourcentage de textes soit disponible sur support lisible par ordinateur (de 3 à $5 \%$ selon les estimations), il n'en reste pas moins que la proportion augmente rapidement et que des outils sont désormais disponibles pour mieux exploiter les textes. Nous n'avons pas l'ambition de brosser un portrait des diverses zones d'intervention des logiciels dans le traitement de la langue des textes et des questions $\mathrm{ni}$ des difficultés à surmonter; nous I'avons fait ailleurs (BertrandGastaldy, 1990a et 1990b). Nous tenterons de montrer comment les tâches de construction de thésaurus, d'indexation et, par le fait même, de repérage peuvent bénéficier de l'assistance de l'ordinateur pour explorer le contenu de bases de données textuelles. Nous prendrons pour cela l'exemple du logiciel SATO qui n'est en aucune façon dédié aux opérations documentaires.

\section{Présentation d'un outil d'aide à I'exploration du contenu des textes : SATO}

\section{Les utilisateurs du logiciel}

Le logiciel SATO (système d'analyse de textes par ordinateur) a été conçu par Jean-Guy Meunier et développé par François Daoust, responsable de l'équipe ITC (Ingénierie cognitive et textuelle) au Centre d'ATO de l'Université du Québec à Montréal. II tourne sur des ordinateurs IBM-PC et compatibles. C'est un logiciel général destiné à un public de chercheurs en sciences humaines et sociales, désireux de faire de l'analyse de contenu pour des raisons fort diverses.

Au ministère de l'Éducation, on I'utilise pour évaluer la lisibilité des textes destinés aux élèves du primaire et du secondaire, en fonction de leur connaissance du vocabulaire et de la syntaxe (Laroche, 1990).

Il a servi, avec Termino, à faire une analyse lexicologique des réponses lors d'une pré-enquête sur "Les lycéens 91 " en France (Le Monde, juin 1991). On peut tout aussi bien $y$ recourir pour les retranscriptions d'entrevues ou d'analyses de protocoles lorsqu'on fait verbaliser un sujet au cours de I'accomplissement d'une tâche. Les psychologues font de même pour analyser et comparer le matériel $\mathrm{d}^{\prime}$ entretien thérapeutique.

Il a été exploité pour l'analyse de textes politiques, notamment par le sociologue Jules Duchastel qui a étudié, entre autres, le discours politique sous le régime Duplessis (Bourque et Duchastel, 1988), ainsi qu'un corpus de dossiers de la cour juvénile de Winnipeg (Duchastel, 1991).

Des linguistes recourent à SATO pour découvrir des structures syntaxiques ou des structures argumentatives dont ils ne posssèdent pas encore de description. Dans sa thèse de doctorat, Anaïd Donabedian s'en est servi pour découvrir des régularités dans l'emploi de l'article en arménien ancien. Monique Lemieux de I'UQAM y trouve une aide précieuse pour l'analyse de la grammaire du moyen français ${ }^{3}$.

Le logiciel sert d'appui aux cogniticiens chargés de dépouiller les textes pour l'extraction des connaissances et la construction de systèmes experts (Paquin et al., 1990), aux terminologues pour l'élaboration de terminologies (Auger, 1990) et finalement, comme nous allons le montrer, à une variété de tâches reliées à la gestion de l'information textuelle en vue d'en faciliter le repérage. D'ailleurs plusieurs des contributions de ce numéro illustrent des réalisations documentaires effectuées à l'aide de SATO.

\section{Les caractéristiques principales}

SATO présente des caractéristiques bien différentes de la plupart des logiciels documentaires commercialisés. Quelques explications préalables sont nécessaires pour apprécier les différentes opérations qu'il permet.

La grande originalité de SATO réside dans le fait qu'il permet d'ajouter des

1. Christopher Locke, "The Dark Side of DIP ", Byte, vol. 16, no. 4 (April 1991), 194.

2. Winfred Schmitz-Esser, "Thesauri Facing New Challenges", International Classification, vol. 17, no. 3-4 (1990), 130.

3. F. Dupuis et al., "Methodological Aspects of the Categorization of a Middle French Corpus with a Computer - Assisted Text Analysis Software (SATO)", Quantitative Linguistics, Université de Trier, Allemagne. (à paraître) 


\section{propriétés aux mots ou segments textuels.}

Au point de départ, tout texte est représenté comme une suite de caractères (y compris le caractère blanc), ce qui est en soi très banal. Restitués à l'écran, ces caractères et les suites de caractères ne peuvent prendre de signification que s'ils sont interprétés par un agent cognitif humain doté de multiples connaissances:

- connaissances de l'alphabet et des divers signes graphiques

- connaissances des morphèmes et des mots de la langue générale, connaissances de la signification des termes particuliers au domaine

- connaissances de la syntaxe

- connaissances du sens commun

- connaissances du domaine luimême

- connaissances de la structure des textes de tel ou tel type (lois, articles scientifiques, rapports de diagnostic médical, etc.)

- connaissances de la signification de certains types de caractères dans le contexte particulier de tel ou tel texte, de tel ou tel ensemble de textes (par exemple, les italiques peuvent indiquer qu'il s'agit d'un titre, d'un mot étranger, les caractères gras sont la marque d'un titre ou d'un sous-titre, les capitales signalent un mot qui est défini dans un glossaire, etc.)

C'est donc l'ensemble de ces connaissances qui interagissent au cours de la lecture pour que du sens soit produit à partir de ce qui n'est que traces sur le papier ou à l'écran. Des opérations cognitives très complexes ont alors lieu qui peuvent consister en généralisation, élimination de détails inutiles, catégorisation, comparaison, reconstruction, etc. Mais la mémoire humaine à court terme a des capacités très limitées, alors que les bases de données peuvent atteindre des gigabytes, d'où l'intérêt de "passer la main " à un logiciel pour des analyses sur des corpus d'envergure.

L'inconvénient c'est que le processeur informatique, contrairement au " processeur humain ", ne connaît rien ou presque rien. Cette métaphore permettra de mieux comprendre ce qu'il faut faire pour qu'un logiciel puisse procéder lui aussi à des catégorisations grammaticales, sémantiques, textuelles, pragmatiques, à des généralisations, à des comparaisons, etc. sur des ensembles de caractères. Des caractéristiques ou propriétés doivent donc être rajoutées, surimposées soit aux mots du lexique, soit aux mots en contexte. Nous en verrons des exemples plus loin. Retenons que SATO conserve en mémoire une représentation fidèle du texte de départ (ce qui permet d'obtenir des références très exactes sur la position des mots à l'intérieur des documents, pages et lignes) et en fait, selon une image empruntée à Maurice Gingras, une photocopie sur laquelle il est possible d'annoter à volonté. Les annotations (qui sont constituées de valeurs de propriétés) peuvent d'ailleurs apparaître en couleurs comme autant de traits de surligneurs.

\section{L'ajout de propriétés peut être:}

1) le résultat d'une opération automatique (les mots en capitales reçoivent la valeur Cap de la propriété Édition, la fréquence devient une propriété numérique des formes);

2) le résultat d'une opération automatique déclenchée par l'analyste (les codes propres à un logiciel de traitement de texte comme le souligné ou les caractères gras peuvent être convertis en valeurs de la propriété Typo; la projection d'un thésaurus ou d'une base de données lexicales sur le lexique résulte en l'attribution à chaque forme des valeurs déclarées)

3) le résultat d'une opération humaine effectuée au cas par cas dans le texte (segmentation et nomination des diverses subdivisions) ou dans le lexique (catégorisation sémantique du vocabulaire hors contexte). A la demande, il est possible de faire passer les propriétés textuelles dans le lexique et vice versa (on parle alors d'héritage de propriété).

L'exemple suivant illustre l'ajout de deux propriétés dans le texte (notice et zone); il s'agit d'une notice de MEDIADOQ dont la structuration en champs a été exploitée et qui a subi un pré-traitement léger, comme on peut le déceler d'après le doublement du point d'abréviation à ne pas confondre avec le point de fin de phrase pour la segmentation automatique en phrases:

\section{*notice $=1$}

*zone=na Do ... \#253

*zone $=$ no A880333

*zone = au Rhéaume, Luc

*zone $=\mathbf{t m}$ Analyse du traitement journalistique de

l'information politique au Québec: le cas du projet de restructuration scolaire de 1982

*zone = so ix, 116, x-xxix f..: tableaux, graph..; $29 \mathrm{~cm}$. - Mémoire (M..A..) - Université Laval, 1984

*zone $=$ re Les formes de dépendance des journalistes à l'égard des sources d'information sont examinées par le biais d'une analyse de contenu de la couverture accordée par la presse écrite francophone au débat entourant le projet de restructuration scolaire proposé par le Gouvernement du Québec en 1982. La première partie porte sur I'information en tant qu'enjeu d'une lutte politique. La deuxième partie expose la méthodologie de la recherche. Cinq journaux quotidiens québécois ont été analysés: La Presse, Le Devoir, Le Soleil, Le Journal de Québec et Le Journal de Montréal. L'analyse porte sur 4 aspects précis de la couverture: les sources d'information, les thèmes abordés, l'approche de traitement et les tendances exprimées. La troisième partie présente les résultats de I'analyse. II ressort que tous les intervenants ont eu un droit de parole dans les pages des journaux, mais que la couverture a été superficielle et axée sur les aspects conflictuels du dossier. La presse ne serait pas en mesure d'expliquer et de vulgariser des problèmes complexes. Par ailleurs les chroniqueurs spécialisés et les journalistes font preuve d'une plus grande autonomie à l'égard des sources d'information que les rédacteurs de nouvelles. Ceux-ci ne font que réagir aux initiatives des sources et adoptent une approche très descriptive; ils se limitent à une fonction de transmission du message des sources. bibliogr..: ff.. x-xiv, (J..C..)

*zone = dep TRAITEMENT DE L'INFORMATION ; PRESSE INFORMATION POLITIOUE ; SOURCE D'INFORMATION *zone =idp SOLEIL, LE ; DEVOIR, LE; PRESSE, LA ; JOURNAL DE Québec, LE; JOURNAL DE Montréal, LE

Comme on peut modifier à volonté les propriétés et leurs valeurs, il n'est pas pénalisant de revenir sur le découpage des textes, au fur et à mesure que les besoins $d^{\prime}$ analyse se précisent.

Du texte, on procède à la génération du lexique dans lequel on peut faire apparaître les propriétés avec leurs valeurs en autant de colonnes.

La fréquence - absolue ou relative - est, tout comme la propriété Édition ou la propriété Alphabet, une propriété prédéfinie. Les autres peuvent être définies au besoin et on peut leur donner n'importe quelle valeur, symbolique ou numérique selon le cas. Voici un extrait de lexique avec affichage de quatre propriétés: I'alphabet (français), la fréquence absolue, la fréquence relative et la (ou les) zones de provenance des formes extraites:

$\begin{array}{cccll}\text { alph } & \text { fréqabs } & \text { fréqrel } & \text { zon } & \text { (lexique) } \\ \mathrm{fr} & 1 & 0.02 & \text { re } & \text { abord } \\ \mathrm{fr} & 1 & 0.02 & \text { re } & \text { aborde } \\ \mathrm{fr} & 1 & 0.02 & \text { re } & \text { abordés } \\ \mathrm{fr} & 1 & 0.02 & \text { so } & \text { abreuver } \\ \mathrm{fr} & 1 & 0.02 & \text { re } & \text { accent } \\ \mathrm{fr} & 1 & 0.02 & \text { re } & \text { acceptation } \\ \mathrm{fr} & 7 & 0.12 & \text { re } & \text { accepter } \\ \mathrm{fr} & 1 & 0.02 & \text { (tm,re,dep,idp) } & \text { accès } \\ \mathrm{fr} & 1 & 0.02 & \text { re } & \text { accord } \\ \mathrm{fr} & 1 & 0.02 & \text { re } & \text { accorde } \\ \mathrm{fr} & 3 & 0.5 & \text { (so,re) } & \text { accordée } \\ \mathrm{fr} & 2 & 0.4 & \text { re } & \text { acheminer } \\ \mathrm{fr} & 10 & 0.18 & \text { re } & \text { acteurs } \\ \mathrm{fr} & 5 & 0.9 & \text { (tm,re) } & \text { activités } \\ \mathrm{fr} & 1 & 0.02 & \text { re } & \text { actuel } \\ \mathrm{fr} & 1 & 0.02 & \text { re } & \text { actuels } \\ \mathrm{fr} & 1 & 0.02 & \text { re } & \text { adhésion } \\ \mathrm{fr} & 1 & 0.02 & \text { re } & \text { administrateurs }\end{array}$




\section{Documentation et bibliothèques}

Avec SATO on peut effectuer des analyses lexico-statistiques. L'exemple suivant indique successivement la moyenne, l'écart-type, la répartition, l'indice de discrimination de Salton et le chi2:

$\begin{array}{crrrrl}\text { Moyenne Écart } & \text { Répart. } & \text { Discri. } & \text { Chi2 } & \\ 0.05 & 0.22 & 5.0 \% & 0.80 & 0.20 & \text { abord } \\ 0.05 & 0.22 & 5.0 \% & 1.02 & 0.26 & \text { accent } \\ 0.05 & 0.22 & 5.0 \% & 0.59 & 0.15 & \text { acceptation } \\ 0.35 & 1.31 & 10.0 \% & 4.33 & 0.55 & \text { accès } \\ 0.05 & 0.22 & 5.0 \% & 0.81 & 0.21 & \text { accord } \\ 0.15 & 0.36 & 15.0 \% & 0.56 & 0.19 & \text { accordée } \\ 0.50 & 1.24 & 15.0 \% & 2.77 & 0.48 & \text { acteurs } \\ 0.25 & 0.89 & 10.0 \% & 2.80 & 0.43 & \text { activités } \\ 0.05 & 0.22 & 5.0 \% & 1.01 & 0.26 & \text { adhésion } \\ 0.05 & 0.22 & 5.0 \% & 0.94 & 0.24 & \text { administrateurs } \\ 0.20 & 0.68 & 10.0 \% & 2.96 & 0.51 & \text { administration } \\ 0.10 & 0.44 & 5.0 \% & 2.22 & 0.40 & \text { agence } \\ 0.55 & 1.36 & 25.0 \% & 3.08 & 0.52 & \text { agences } \\ 0.05 & 0.22 & 5.0 \% & 0.59 & 0.15 & \text { aide } \\ 0.05 & 0.22 & 5.0 \% & 0.80 & 0.20 & \text { ailleurs } \\ 0.05 & 0.22 & 5.0 \% & 1.02 & 0.26 & \text { alarmiste } \\ 0.05 & 0.22 & 5.0 \% & 1.09 & 0.28 & \text { alliances } \\ 0.10 & 0.44 & 5.0 \% & 1.76 & 0.32 & \text { américaines }\end{array}$

Une seule commande suffit pour obtenir la participation d'une forme dans les textes ou sous-textes d'un corpus:

Participation de information
$\begin{aligned} & \text { nombre de lexèmes: } \\ & \text { domaine } \quad \text { vocabulaire/domaine }\end{aligned}$
$\begin{array}{lcc}\text { fréqtot } & \text { domaine/texte }\end{array}$

Cet affichage indique que le mot information apparaît 101 fois dans le corpus et constitue $1.80 \%$ de l'ensemble du texte.

On peut travailler séparément sur le lexique - et le corpus - de chaque langue, dans le cas de textes multilingues (maximum de quatre langues).

On peut connaître les formes qui distinguent le plus un texte d'un autre, un champ d'un autre dans les notices bibliographiques, grâce à la commande Distance. Ainsi, à partir des subdivisions introduites par une propriété textuelle, on peut comparer, par exemple, le vocabulaire de deux bases de données ou celui des différentes zones des notices, celui des titres et des sous-titres, celui des introductions et celui des conclusions, celui d'un chapitre de livre par rapport à un autre (ou à l'ensemble des autres, comme dans l'exemple suivant):

\footnotetext{
fréq- fréq- autr- expli- cumul (lexique)

tot chap chap que

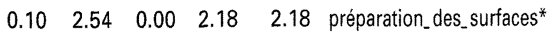

$\begin{array}{llllll}0.05 & 1.69 & 0.00 & 1.94 & 4.12 & \text { cuivrage* }\end{array}$

$\begin{array}{lllll}0.05 & 1.69 & 0.00 & 1.94 & 6.06 \\ \text { décapage* }\end{array}$
}

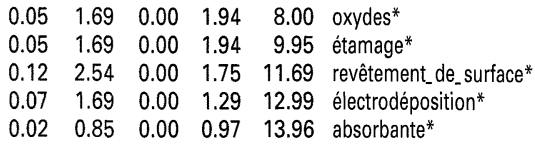

...

Un indice de lisibilité est fourni à la demande. Il est calculé selon la formule de Gunning (dont on conteste cependant la validité pour le français) et tient compte de la longueur des phrases et de la proportion de mots longs (9 caractères et plus). Mais les chercheurs du ministère de l'Éducation travaillent à d'autres indices qui sont fonction des capacités de compréhension des élèves selon leur âge:

\section{1 mots de 1 car. (4\%) 619 mots de 3 car. (13\%) 286 mots de 5 car. $(6 \%)$ 430 mots de 7 car. $(9 \%)$ 267 mots de 9 car. $(5 \%)$ 203 mots de 11 car. $(4 \%)$ 80 mots de 13 car. $(2 \%)$ 31 mots de 15 car. (1\%) 2 mots de 17 car. $(0 \%)$ 2 mots de 19 car. $10 \%)$ \\ 3 mots de 21 à 25 car. $(0 \%)$ 0 mots de plus de 30 car. $10 \%)$ \\ nombre de mots ........ 4923 nombre de phrases .... 318 nombre de paragraphes ..1 \\ 1372 mots de 2 car. $(28 \%)$ 360 mots de 4 car. $(7 \%)$ 390 mots de 6 car. $(8 \%)$ 298 mots de 8 car. $(6 \%)$ 229 mots de 10 car. $(5 \%)$ 96 mots de 12 car. $(2 \%)$ 43 mots de 14 car. (1\%) 10 mots de 16 car. $10 \%)$ 1 mots de18 car. (0\%) 0 mots de 20 car. $10 \%)$ 0 mots de 26 à 30 car. $(0 \%)$ \\ longueur moy. : $5.3 \mathrm{car}$. longueur moy. : 15.5 mots longueur moy. : 4923.0 \\ pourcentage de mots de 9 lettres et plus : $20 \%$ \\ indice de lisibilité de Gunning: 14.0}

Nous arrêterons là notre présentation générale. D'autres fonctionnalités seront expliquées au fur et à mesure des besoins.

Depuis trois ans environ, nous avons testé, dans des cours et des projets de recherche, les capacités de SATO à traiter les textes pleins ou les textes réduits (notices bibliographiques et analytiques) pour différentes fonctions documentaires ${ }^{4}$. Nous examinerons d'abord comment SATO peut aider à contrôler et structurer un vocabulaire de domaine.

Les opérations que nous allons présenter supposent que les textes ont été soumis au préalable en format ASCII, avec au minimum la déclaration de la propriété Alphabet et un titre.

\section{Construction de thésaurus}

Effectuée de façon traditionnelle, soit à partir des résultats de l'indexation manuelle des textes (méthode a posteriori), soit en consultant les ouvrages terminologiques ou lexicographiques ainsi que les experts du domaine (méthode a priori), la construction de thésaurus est une tâche trop onéreuse, insuffisamment rigoureuse et le résultat trop décalé par rapport aux besoins pour que les organisations y accordent volontiers les ressources nécessaires.

\section{La nécessité du contrôle du vocabulaire}

Pourtant les résultats des évaluations du repérage en plein texte prouvent la nécessité d'une telle opération. Le taux de rappel est bas si un réseau de relations ne vient pas suggérer des moyens d'élargir les stratégies par des termes génériques ou par d'autres termes spécifiques d'une même classe. Par exemple, si l'on veut récupérer tout ce qui traite des crucifères cultivées, il faut savoir que l'information peut être dispersée sous chacun des termes spécifiques: choux, choux-fleurs, brocolis, choux-deBruxelles, choux chinois, rutabagas, radis. C'est ainsi que les évaluations de Blair (1986) et Blair et Maron (1985) sur le système STAIRS/TLS révèlent un taux de rappel de $20 \%$ seulement. L'étude de Balcer et Gonin (1979) avait abouti à un taux de $41,3 \%$, performance peu reluisante que les auteurs attribuaient à l'absence de renvois et par conséquent à des stratégies trop limitées. En plein texte, le taux de précision peut, à force de reformulations de la stratégie de recherche, atteindre un score très satisfaisant, mais c'est souvent au prix de beaucoup d'effort et de temps. D'après Garson et Love (1985), une référence pertinente dans ACS Journals Online, interrogée sur le serveur BRS, coûte en moyenne le double d'une notice trouvée dans Chemical Abstracts sur le serveur DIALOG. Tenopir (1985) a obtenu le même genre de résultats dans une étude qui comparait la recherche sur le plein texte et la recherche sur les résumés et les descripteurs, dans Harvard Business Online.

4. Nous avons travaillé en collaboration avec les chercheurs du Centre d'ATO et plusieurs professionnels de la fonction publique québécoise. Plusieurs contrats ou subventions ont " alimenté " nos recherches: $\mathrm{CRSH}$ (Centre de recherche en sciences humaines) interne, contrats du ministère des Communications, subvention du CEFRIO (Centre francophone de recherche en informatisation des organisations). 
L'informatisation des opérations liées à la construction des thésaurus

L'automatisation a surtout touché jusqu'à présent la gestion des résultats de la collecte, du contrôle et de la structuration des termes. De nombreux logiciels existent qui assurent la validation, la réciprocité des relations, la mise à jour du contenu des thésaurus, l'édition, etc. en conformité avec les normes nationales et internationales.

Depuis longtemps déjà, des méthodes statistiques ont été testées pour extraire le "vocabulaire" de corpus textuels et en construire une représentation structurée selon des algorithmes de classification automatique. On pense surtout aux travaux de Salton (Salton, 1971) et de SparckJones (1971)et à tout le débat sur les "thésaurus de recherche" (Lancaster, 1977; Bertrand-Gastaldy, 1984; Richer, 1986). L'intérêt des résultats de ces méthodes est cependant limité à certains types d'utilisation: les représentations orientent les utilisateurs qui connaissent peu le contenu de la base de données interrogée ou bien offrent des suggestions de "mots" liés d'une certaine manière à ceux qui figurent dans les stratégies de recherche, mais I'interprétation de la nature des liens reste à la charge des utilisateurs. Couplés à un système d'inférences chargées d'élargir ou de restreindre les stratégies, de tels outils risquent de diriger la recherche dans toutes sortes de direction. D'autre part les unités de représentation extraites sont hétérogènes: ce sont tantôt des "mots", tantôt des lexies, tantôt des termes, tantôt des radicaux.

L'opération de sélection et de structuration fine des termes d'un domaine, affaire de spécialistes qu'ils soient indexeurs, terminologues ou "ingénieurs cogniticiens ", souffre encore de la rareté d'outils adéquats pour l'assister, ce qui a fait écrire à Ranjard (1991): "[...] force est de reconnaître que les outils de gestion de thésaurus n'aident en rien à la conception des vocabulaires contrôlés. [...]", ce qui contraint les documentalistes à attendre "que les outils informatiques $d$ 'analyse de contenu des textes soient à la portée de tous ".

\section{L'aide apportée par SATO dans les différentes étapes d'élaboration d'un thésaurus}

SATO peut apporter une aide non négligeable dans la conception de vocabulaires de domaines, comme nous allons tenter de l'illustrer.

\section{Choix du moment opportun pour entreprendre la construction d'un thésaurus}

Mentionnons que, par ses fonctions statistiques, SATO fournit des indices utiles pour qui doit décider de l'opportunité d'entreprendre la construction de thésaurus. La fréquence moyenne des formes, ou taux de répétitivité, la proportion des mots de fréquence faible $(1,2$ et 3 ) et le calcul des formes nouvelles à chaque ajout d'une certaine quantité de textes permettent d'apprécier le plafonnement du renouvellement du vocabulaire, moment qu'il est sage d'attendre si l'on ne veut pas faire face à de nombreux problèmes de mise à jour.

\section{Extraction du vocabulaire}

\section{Les formes simples}

Comme tout autre logiciel, SATO fournit le lexique des formes simples contenues dans la base de données. II tient compte de tous les caractères et n'élimine ni les signes de ponctuation ni les formes fonctionnelles, sauf si on le demande. Nous verrons plus loin l'intérêt des les conserver.

\section{La catégorisation grammaticale des formes simples}

SATO permet d'affecter à chaque forme du lexique la ou les catégories grammaticales qu'elle peut prendre hors contexte. L'affectation s'effectue de façon automatique, avec la procédure Dogramr à partir de la consultation de bases de données lexicales. Notons qu'on peut recourir aux sources lexicographiques ou terminologiques de son choix. Le résultat se présente ainsi :

$\begin{array}{cl}\text { fréq } & \text { gramr } \\ 1 & \text { nomp } \\ 2 & \text { (adj,nomc) } \\ 2 & \text { (adj,nomc) } \\ 1 & \text { (adj,nomc) } \\ 1 & \text { nomc }\end{array}$

$\begin{array}{ll}v_{-} \text {conj } & \text { caractérise } \\ \text { (adj,nomc) } & \text { caractéristiques } \\ \text { nomc } & \text { cas } \\ \text { nomc } & \text { catégories } \\ \text { adj } & \text { catholique } \\ \text { adj } & \text { catholiques } \\ \text { nomc } & \text { causes } \\ \text { (détdém,p_dém) } & \text { ce }\end{array}$

Une simple commande permet de connaître le pourcentage de formes qui sont à la fois verbe et nom, nom et adjectif, etc. :

\begin{tabular}{|c|c|c|}
\hline nombre & pourcent & symbole \\
\hline 449 & $31.58 \%$ & nomc \\
\hline 136 & $9.56 \%$ & (adj,nomc) \\
\hline 133 & $9.35 \%$ & détnum \\
\hline 1278 & $93 \%$ & nomp \\
\hline 94 & $6.61 \%$ & adj \\
\hline 93 & $6.54 \%$ & $v_{-}$conj \\
\hline
\end{tabular}

On peut faire afficher la liste des noms, des verbes, des formes qui n'ont reçu aucune valeur et, si on le souhaite, procéder à des ajouts et à des corrections dans le lexique ou en contexte.

Pour les formes nouvelles, celles qui ne figurent pas dans les sources consultées, il faut les catégoriser à la main. On peut le faire en bloc dans certains cas, d'après le suffixe (les mots qui se terminent par les caractères able ou ables sont en général des adjectifs, à part quelques exceptions comme table) ou au cas par cas, selon l'ampleur de la mise à jour nécessaire et la diversité des formes. Le lexique d'une base de données avec les valeurs de catégorie grammaticale peut être sauvegardé sous forme de dictionnaire et être réutilisé au besoin.

\section{Les expressions complexes}

L'ajout de valeurs de propriété grammaticale rend possible la recherche dans le texte de toute séquence de valeurs comme:

Nom commun + nom propre: île Dupas, lac Saint-Pierre, rue Ste-Catherine

Nom commun + adjectif ou participe passé: acide chromique, chaux hydratée

Nom commun + de ou d'+ nom commun ou nom propre: cours d'eau, plan d'urbanisme, village de Bernierville

Nom commun + de ou d' + nom commun + adjectif ou participe passé: bureau d'audiences publiques,

température d'ébullition normale

Nom commun + adjectif ou participe passé + de ou $d^{\prime}$

+ nom commun ou nom propre: agents chimiques de coagulation etc. 
Cette méthode dite de patrons catégoriels s'assortit d'une procédure exécutable (I'équivalent d'une macrocommande dans WordPerfect) Marqterm qui "bloque" au moyen d'un trait de soulignement les différents éléments de l'expression complexe, de telle sorte qu'elle soit désormais considérée comme une simple unité lexicale. Selon le degré d'exhaustivité cherché, divers patrons peuvent être spécifiés qui correspondent aux modes les plus courants de formation des lexies complexes. Le lexique issu de la procédure ressemble à ceci :

\author{
fonction_publique \\ hautes_eaux_printanières_moyennes \\ métaux_sous_forme_de_carbonate \\ Office_des_ressources_humaines \\ personnel_engagé_à_honoraires
}

On constate donc l'avantage d'un logiciel qui n'élimine pas les "motsoutils " par un anti-dictionnaire, puisque les prépositions jouent un rôle important dans la formation des lexies complexes, et qui permet de formuler des stratégies de recherche incluant autant les chaînes de caractères (de, $\left.d^{\prime}\right)$ que leurs valeurs de propriétés (préposition, par exemple). II s'agit d'une fouille exhaustive et systématique qui n'oblige pas à repérer et à énumérer auparavant les mots susceptibles d'entrer dans la composition de termes du domaine. Les opérations effectuées sur les propriétés constituent un raccourci pour traiter l'ensemble des unités pertinentes.

Pour les différentes variantes possibles de la procédure et sur ses limites évidentes (en particulier le bruit généré par la non-désambiguïsation en contexte des valeurs multiples), le lecteur pourra se reporter au chapitre 9 de l'ouvrage de Bertrand-Gastaldy (1992).

Ce dispositif aide donc à repérer les fameux multitermes qui sont un véritable casse-tête pour l'établissement des thésaurus. On évalue, en effet, que jusqu'à $80 \%$ des concepts d'un domaine sont exprimés par ce que les linguistes appellent des termes complexes $^{5}$.

\section{Les termes du domaine}

Une fois la liste des expressions complexes épurées de ses scories évidentes et une fois prises les décisions délicates de la décomposition en unités plus petites, il reste à détermi- ner ce qui correspond à un terme du domaine, c'est-à-dire à un sousensemble du lexique faisant appel à la notion d'usage au sein d'une communauté scientifique donnée.

Le choix définitif des termes du domaine, qu'ils soient simples (unitermes) ou complexes (multitermes), demeure l'apanage des experts. Nous pouvons profiter de diverses fonctionnalités de SATO pour offrir à ces experts une liste exhaustive, mais ordonnée selon un ordre probable de pertinence décroissante. La pondération part de différentes propriétés des unités lexicales, fréquentielles, typographiques, textuelles, etc. Les propriétés et les valeurs qui entrent en ligne de compte ne sont pas déterminées d'avance; elles sont laissées à la discrétion des concepteurs du thésaurus, ce qui permet de les ajuster aux particularités des corpus.

Si, dans un corpus, tous les mots importants sont en gras ou en italiques, on peut décider d'accorder un certain poids à cette propriété. En outre, les titres et les sous-titres, les légendes de tableaux, les tables des matières et les index constituent de bons réservoirs de termes et l'on a vu qu'on peut caractériser explicitement ces différents éléments. On pourrait même décider d'accorder un poids supérieur aux unités lexicales qui figurent dans les deux premières et les deux dernières phrases de chaque paragraphe (cette possibilité a été rajoutée à notre demande, dans un logiciel qui peut travailler en amont de SATO, ICON par un des collaborateurs dans deux de nos projets). On peut aussi pondérer non pas à partir de la fréquence, mais de la valeur discriminante de Salton implantée depuis peu dans SATO ou bien en fonction du chi2 qui est disponible depuis longtemps avec la commande Distance.

Voici illustrés les résultats de la procédure qui a consisté à ajouter à la fréquence: + 14 aux termes complexes, + 10 aux noms communs, + 2 aux verbes et +1 aux adjectifs et à ajouter +5 si les formes se trouvent dans la macrostructure des textes (titres, sous-titres, etc.):

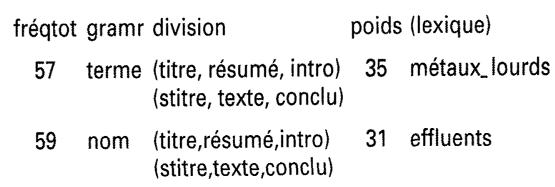
6 terme (résumé,intro) (texte)
16 terme (résumé,texte)
30 échange_ionique
11 nom (résumé,intro)
30 osmose_inverse (stitre,texte)
20 cémentation

Ce qu'on peut constater donc, c'est l'éventail et la complémentarité des approches possibles pour mieux cerner le vocabulaire du domaine. Le logiciel offre des "prises de vue" multiples sur le vocabulaire. Un faisceau d'indices est ainsi disponible pour assister le choix.

Une fois la liste éditée, on peut encore recourir à SATO pour enregistrer le jugement de plusieurs experts et procéder à l'élimination des unités non pertinentes sur la base d'un simple calcul. Voici un exemple qui montre l'avis de trois spécialistes:

$$
\begin{aligned}
& \text { fréqtot poids expert1 expert2 expert3 (lexique) } \\
& \begin{array}{rrrrrl}
57 & 35 & \text { oui } & \text { oui } & \text { oui métaux_lourds } \\
59 & 31 & \text { oui } & \text { oui } & \text { oui } \\
6 & 30 & \text { oui } & \text { oui } & \text { oui échants } \\
16 & 30 & \text { oui } & \text { oui } & \text { oui } & \text { osmose_ingerse } \\
\ldots & & & & & \\
1 & 14 & \text { oui } & \text { non } & \text { non } & \text { contrôle_simple } \\
\ldots & & & & & \\
1 & 14 & \text { non } & \text { non } & \text { non demande_faible } \\
1 & 14 & \text { non } & \text { non } & \text { non demande_forte }
\end{array}
\end{aligned}
$$

Après le choix définitif, il est possible de sauvegarder la liste des termes du domaine sous forme d'un dictionnaire qui pourra être réutilisé par la suite sur tout nouveau texte entré dans la base de données.

\section{Contrôle du vocabulaire}

Dans les bases de données en plein texte, on peut entendre par contrôle tout ce qui contribue à favoriser l'univocité du vocabulaire: le regroupement a posteriori des variantes flexionnelles autour d'une forme canonique, la mise en relation des différentes graphies d'un même terme, la mise en évidence de la polysémie en autant qu'elle existe dans un domaine de spécialité, au moyen de définitions et de contextes, et finalement le regroupement de synonymes ou quasi-synonymes. Le contrôle est d'autant plus nécessaire

5. Jean-Claude Boulanger, "Le statut du syntagme dans les dictionnaires généraux monolingues", Meta, vol. 34, no 3 (septembre 1989), 361. 
que les unités sont plus nombreuses et plus variées.

\section{$\underline{\text { Les variantes flexionnelles }}$}

Une des lacunes de SATO est qu'il ne possède pas d'analyseur syntaxique; il est donc impuissant à résoudre les problèmes d'identité (homographie) que l'on trouve par exemple dans:

\footnotetext{
lit___ > sorte de meuble (nom commun, masculin, singulier)

lit___ > une des formes possibles de lire (verbe)

avions_ > appareil de locomotion aérienne (nom commun, masculin, pluriel)

avions_ > une des formes possibles de avoir (verbe)
}

ainsi que les problèmes d'altérité, $c^{\prime}$ est-à-dire du rattachement de deux chaînes distinctes à un même lexème, comme dans:

lits $\_$lit $\_$lit (nom commun, masculin)
lit__ $\_$lire (verbe)

Il existe encore peu de logiciels qui procèdent à une lemmatisation automatique car, pour cela, il faut reconnaître la fonction syntaxique du mot en contexte, lui attribuer une valeur grammaticale unique et finalement renvoyer la forme flexionnelle à la forme canonique. On notera cependant que, dans la phase 2 du projet Delta du ministère des Communications du Québec, il est prévu que le logiciel Termino, mis au point par l'équipe RDLC (Recherche et développement en linguistique computationnelle) du Centre d'ATO pour le dépistage de lexies complexes à l'Office de la langue française, fournisse à SATO les résultats de son analyse syntaxique.

En attendant, il est toujours possible d'effectuer ce renvoi pour toutes les formes qui, hors contexte, n'ont qu'une valeur, c'est-à-dire sont uniquement noms, uniquement adjectifs ou uniquement verbes, mais il s'agit d'une solution " boîteuse " parce qu'incomplète.

Formes équivalentes: graphies différentes, abréviations, sigles et acronymes

Par rapport aux autres logiciels documentaires, l'aide originale qu'apporte SATO consiste dans le repérage des régularités d'ordre typographique ou linguistique susceptibles de marquer une variante graphique, une abréviation, un sigle ou un acronyme. La plupart du temps, les sigles et acronymes sont inscrits en lettres capitales et sont accompagnés, lors de leur première mention, de la forme pleine équivalente. Une parenthèse, parfois des tirets, distingue les deux. On peut se faire une idée du genre de stratégie de recherche et de réponses fournies d'après cet exemple où l'on cherche toute séquence qui comporterait une parenthèse ouvrante suivie de n'importe quelle chaîne de caractères écrite en capitales et où l'on demande d'écrire le contexte (pour alléger la présentation, on a supprimé les références):

\section{Concordance stricte $\backslash(\$$ édit $=$ cap}

Écrire concordance*

La matrice du polymère de l'échangeur peut être un copolymère_de_styrène et de benzène_divinylique (DVB), phénol et formaldehyde ou des polymères_naturels, tels que le charbon_sulfoné.

le thénoyltrifluoroacétone (TTA) possède une sélectivité très élevée pour le cuivre, à pH très bas;

On constate une fois de plus l'intérêt d'un logiciel qui permet de fouiller non seulement les mots, mais aussi n'importe quel caractère comme un signe de ponctuation.

\section{Synonymie}

La stratégie utilisée précédemment peut être adaptée pour repérer des synonymes. Évidemment, les réponses du système vont comporter beaucoup de bruit, mais on sera sûr de l'exhaustivité de la fouille en autant qu'on aura su répertorier les divers marqueurs de synonymie: et, comme, ou, est désigné(e) par, sont désigné(e)s par, etc. II restera à user de ses connaissances du domaine - et souvent du simple bon sens - pour ne conserver que les passages pertinents. L'établissement de relations de synonymie est une opération sémantique qu'un ordinateur ne peut effectuer. On sait que la synonymie dépend beaucoup du contexte et de la possibilité de visionner ces contextes (qui, soit dit en passant, sont paramétrables dans SATO). Voici un exemple de résultats:

Le revêtement de surface est désigné par les termes d'électrodéposition, de galvanotechnique ou de galvanostégie.

Nous verrons plus loin comment enregistrer les relations entre formes. Nous allons continuer, pour le moment, à illustrer l'aide que le logiciel peut apporter dans le dépistage de ces relations.

\section{Les définitions}

De la même façon, on peut repérer des définitions qui pourront donner lieu à des notes d'application dans le thésaurus. Selon les régularités des textes, on pourra chercher une séquence de deux-points et de guillemets ouvrants, ou bien des expressions comme définir, entendre par, etc.

"emploi excédentaire": un emploi autorisé provisoirement en surplus des effectifs réguliers

La complexation se définit comme la formation d'un composé_complexe par un agent_complexant_ou_chelatant

Le revêtement_de_surface est désigné par les termes_d'_électrodéposition, de galvanotechnique ou de galvanostégie. On peut le définir comme l'action de déposer, par voie_électrique, un métal ou un alliage sur un autre métal ou des plastiques.

\section{Structuration du vocabulaire}

Pour ordonner la multiplicité des unités lexicales recueillies et fournir une image cohérente du domaine, on procède habituellement à plusieurs types de regroupements qui en facilitent la consultation et fournissent des suggestions pour élargir ou rétrécir les stratégies de recherche.

\section{Regroupement par thèmes}

Pour identifier les grandes thématiques, il est possible de s'appuyer sur un simple affichage du lexique. Dans la zone des fréquences élevées, là où I'on retrouve habituellement les formes grammaticales courtes, se détachent des formes longues, habituellement des noms qui correspondent à ce que l'on appelle les motsthèmes. Ensuite, à l'aide de la commande Tamiser, on fait afficher les mots qui cooccurrent le plus souvent avec ces mots-thèmes et l'on obtient une première organisation. Voici les fréquences les plus élevées d'un corpus:

\begin{tabular}{|c|c|c|c|}
\hline \multicolumn{4}{|c|}{ Écrire lexique $\$$ tri fréqtot } \\
\hline alphabet & fréqtot & (lexique) & \\
\hline $\mathrm{fr}$ & 902 & de & \\
\hline $\mathrm{fr}$ & 695 & . & \\
\hline $\mathrm{fr}$ & 693 & , & \\
\hline$\ldots$ & 148 & métaux & $<-$ \\
\hline $\mathrm{fr}$ & 101 & $\mathrm{pH}$ & $<$ \\
\hline$\ldots$ & 81 & précipitation & $<$ \\
\hline $\mathrm{fr}$ & 76 & effluents & $<$ \\
\hline
\end{tabular}




\section{Documentation et bibliothèques}

$\begin{array}{llll}\mathrm{fr} & 76 & \text { élimination } & < \\ \mathrm{fr} & 73 & \text { a } & \\ \mathrm{fr} & 73 & \text { qui } & \\ \mathrm{fr} & 70 & \text { concentration } & < \\ \mathrm{fr} & 69 & \text { procédé } & < \\ \ldots \ldots & & & \\ \mathrm{fr} & 66 & \text { cuivre } & < \\ \mathrm{fr} & 63 & \text { lourds } & \\ \ldots & & & \\ \mathrm{fr} & 59 & \text { traitement } & \\ \mathrm{fr} & 56 & \text { ions } & < \\ \ldots & & & \\ \mathrm{fr} & 47 & \text { effluent } & \\ \mathrm{fr} & 47 & \text { hydroxyde } & \\ \mathrm{fr} & 44 & \text { forme } & \\ \mathrm{fr} & 44 & \text { il } & \\ \mathrm{fr} & 42 & \text { métal } & \end{array}$

\section{$\underline{\text { Regroupement par facettes }}$}

La subdivision du corpus en plusieurs thèmes ou domaines (domaine juridique, administratif, économique, social, etc.) n'est pas suffisante pour introduire une structure. II faut souvent chercher les différentes facettes des domaines en question.

Les suffixes peuvent servir à détecter des formes dont les membres partagent une caractéristique commune. Par exemple, les formes qui finissent par -ité expriment souvent des qualités, celles en -ation correspondent souvent à des actions ou à des processus.

$\begin{array}{ll}\text { acidité } & \begin{array}{l}\text { accumulation } \\ \text { anodisation }\end{array} \\ \text { alcalinité } & \text { augmentanchéité } \\ \text { humidité } & \text { automatisation } \\ \text { polarité } & \text { caractérisation } \\ \text { réactivité } & \text { cémentation } \\ \text { solubilité } & \text { centrifugation } \\ \text { stabilité } & \text { chloration } \\ \text { toxicité } & \text { clarification } \\ \ldots & \ldots\end{array}$

La recherche de concordances de termes qui peuvent désigner explicitement cette caractéristique, par exemple procédés ou processus, complète utilement la fouille:

procédés

$\# 2{ }^{*}$ page $=$ doc $1 / 3 / 10 \ldots *$ page $=$ doc $1 / 3 / 15 / 6$

* divis = résumé La première regroupe les procédés courants pour éliminer les métaux_lourds des effluents, tels: la précipitation_chimique_sous_forme_d'_hydroxydes, de carbonates, de sulfures, de phosphates ou de métal_élémentaire; la coprécipitation; la séparation_solide-liquide_par_filtration, flottation, sédimentation ou centrifugation; la coagulation-floculation, et le traitement_biologique.

processus

$\# 10{ }^{*}$ page $=$ doc $1 / 8 / 17 / 6 \ldots{ }^{*}$ page $=$ doc $1 / 8 / 21 / 2$

* divis =intro Ces processus sont: la précipitation_chimique, la coagulation-floculation, l'échangeionique, l'extraction_par_solvant, la cémentation, la complexation, les traitements électrochimiques ou biologiques, l'évaporation et la séparation_par_membranes.

\section{Regroupement par familles de mots}

Pour contrer la dispersion de l'information due à la variété de la nature grammaticale des mots beaucoup plus grande dans un corpus en plein texte que dans une liste de mots clés contrôlés où ne sont conservés en général que les noms, il est commode de procéder à des regroupements autour d'un même radical. Du même coup, on contrôle partiellement certaines conséquences fâcheuses de la synonymie phrastique. Cette opération s'effectue à partir de la troncature à droite, qui peut pallier en même temps l'absence de lemmatisation, et aussi à partir de la lemmatisation à gauche si l'on veut établir des listes de mots de la même famille. Ainsi avec la stratégie Iplant \$, on récupère:

\section{planter}

plants

transplant

transplants

transplanter

transplantation

replanter

Le rattachement des mots de même famille n'est malheureusement pas aussi automatique qu'il y paraît, à cause des modifications du radical (voir, vue), de la formation savante ou populaire (caprin, chèvre) de l'origine latine ou grecque du radical (aquatique, hydrique) et de la différenciation sémantique des dérivés (receveur, récepteur).

Voici des passages qui mentionnent, sous différentes formes, la notion de récupération des métaux:

\section{Concordance libre récup $\$$ méta $\$$ \\ récupéré \\ La solution peut être réutilisée et le métal éliminé est récupéré. \\ récupérés}

La quantité des métaux récupérés est trop petite pour intéresser les principales compagnies qui les utilisent.

\section{récupèrent}

Les procédés de séparation physique, tels la précipitation, la filtration, la floculation et l'évaporation, récupèrent les métaux sous forme d'oxydes, d'hydroxydes ou de sels.

\section{récupérer}

L'objectif de ce rapport est de faire une revue critique de la littérature sur les procédés en usage et des nouvelles technologies qui permettent d'éliminer, de récupérer et de recycler les métaux lourds contenus dans les effluents industriels. récupération

La récupération de chacun des métaux n'est pas toujours possible.

\section{Relations hiérarchiques}

On sait que les relations hiérarchiques constituent l'ossature du thésaurus. Leur dépistage s'effectue au moyen d'expressions comme: est un(e), sont tel(le)(s) le, la, tel(le)(s) que ou bien par une stratégie qui recherche tout nom précédant un caractère de ponctuation comme la parenthèse ouvrante ou encore les deux-points, caractère luimême suivi d'un ou plusieurs noms (c'est ce qu'on appelle une tournure cataphorique), avec, le cas échéant, une fermeture de la parenthèse. Voici un exemple de stratégie de recherche:

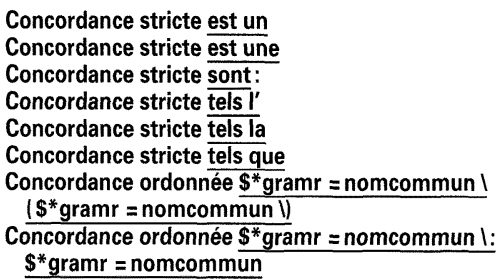

avec des extraits de résultats:

L'effluent est passé en premier à travers une résine cationique pour éliminer les cations tels que le $\mathrm{Fe}$, le $\mathrm{Cu}$, le $\mathrm{Zn}$, le $\mathrm{Ni}$ et le $\mathrm{Cr} 3+$.

Les produits_chimiques qui sont utilisés fréquemment pour précipiter les métaux lourds_sous_forme_ $d$ ' hydroxydes sont la chaux, la soude, la soude_caustique et l'oxyde_de_magnésium

Le procédé est d'autant plus intéressant qu'il y a présence de métaux_précieux (or, argent, platine) dans les effluents.

L'utilisation de Fes comme co-précipiteur des métaux_lourds (cuivre, cadmium, nickel, chrome et zinc) s'avère avantageuse comparativement aux hydroxides.

toutes les crucifères cultivées: chou, chou-fleur, brocoli, chou de Bruxelles, chou chinois, rutabaga et radis

De plus, un paradigme peut être constitué autour d'un nom suivi de ses différentes déterminations comme ceci :

Congé

Congé à temps plein

Congé à traitement différé

Congé de maladie

Congé de préretraite

Congé de maternité

Congé hebdomadaire

Congé partiel

Congé pour adoption

Congé pour affaires judiciaires

Congé pour événements familiaux

Congé pour responsabilités parentales

Congé sabbatique

Congé sans traitement 
On peut alors obtenir des sousensembles regroupant les concepts par une de leurs caractéristiques communes. Le choix des caractéristiques se fait de façon empirique, $d^{\prime}$ après la liste des termes recueillis dans le corpus:

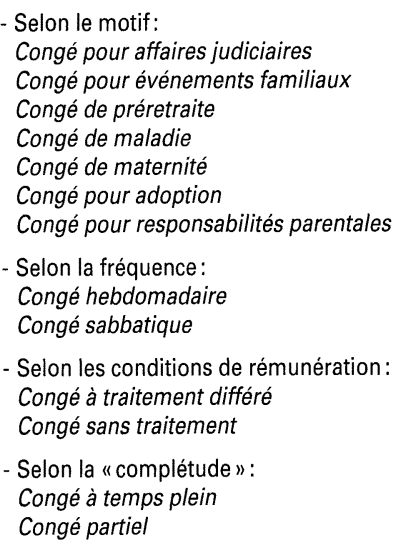

- Des termes spécifiques peuvent provenir d'une combinaison de caractéristiques:

Congé partiel sans traitement

Quant aux relations partitives, qui sont rangées avec les relations hiérarchiques dans les normes de thésaurus, elles sont détectées par des expressions comme partie de, membre de... L'article de Serge Houde dans ce même numéro montre l'intérêt de l'exploitation de tournures de ce genre dans les définitions de dictionnaires électroniques.

\section{Recherche de termes associés}

Les relations associatives, parce qu'elles sont souvent établies sans grande rigueur, ont déjà été qualifiées de véritable fourre-tout. Elles regroupent des termes qui, dans les énoncés textuels, entretiennent des relations lexico-syntaxiques, du type action objet de l'action - instrument de I'action comme Communication, Communiqué, Radio, des relations entre déterminé et déterminant comme Permis d'absence, Absence, des relations entre termes de sens voisin comme Efficacité, Efficience, des relations entre désignation du concept et propriétés du concept comme Sol, Humidité. II a été suggéré à plusieurs reprises de les remplacer par des relations de co-occurrence non étiquetées, mais établies de façon plus systématique et reflétant mieux le contenu réel de la base de données à interroger.
Dans SATO, la commande Tamiser filtre, pour une forme donnée (chaux vive dans l'exemple ci-dessous), les formes utilisées dans le même contexte, la phrase par exemple, et indique la fréquence de cooccurrence:

$\begin{array}{cl}\text { freqass } & \text { (lexique) } \\ 4 & \text { chaux_vive } \\ 3 & \text { chaux_hydratée } \\ 2 & \text { équipements_spéciaux } \\ 2 & \text { nécessite } \\ 2 & \text { utiliser } \\ 1 & \text { brûlures } \\ 1 & \text { cadmium } \\ 1 & \text { causer }\end{array}$

II est possible de préciser, pour la forme spécifiée, la nature grammaticale des mots associés: les verbes seulement, ou les noms et adjectifs, par exemple. Le texte de Patrick Cossette dans cette même livraison de la revue illustre I'utilisation de cette méthode pour établir des classes de mots propres à un domaine.

\section{Enregistrement et gestion des relations entre mots}

Les différents types de relations sont considérés comme des propriétés et les mots reliés sont autant de valeurs de propriété. Ainsi peut-on avoir:

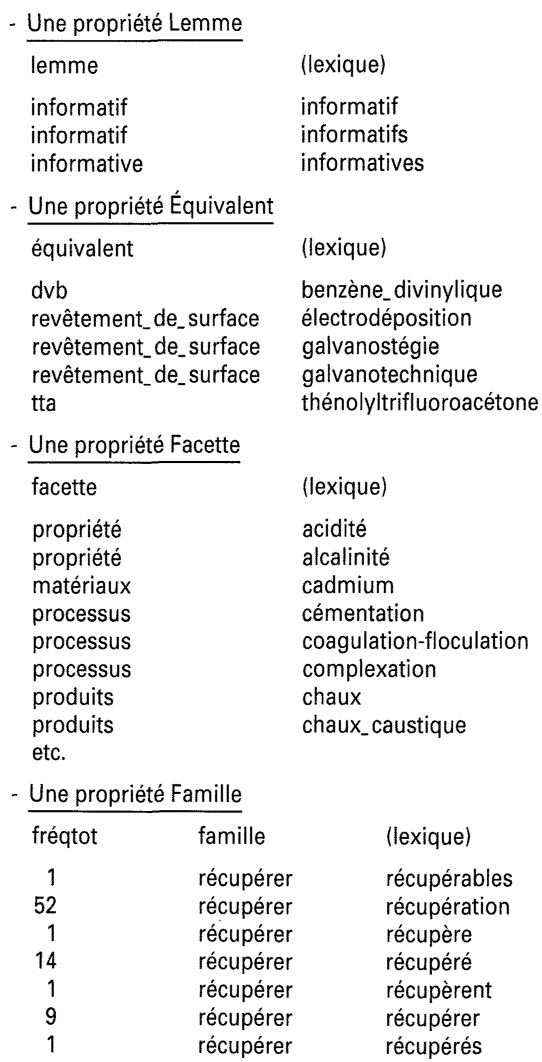

$\begin{array}{ll}\begin{array}{ll}\text { - Une propriété Hiérarchie } \\ \text { hiérarchie }\end{array} & \text { (lexique) } \\ \text { (métaux, métaux_précieux) } & \text { argent } \\ \text { (métaux, métaux_lourds) } & \text { cadmium } \\ \text { (métaux, métaux_lourds) } & \text { chrome } \\ \text { (métaux, métaux_lourds) } & \text { cuivre } \\ \text { (métaux) } & \text { métaux } \\ \text { (métaux, métaux_lourds) } & \text { métaux_lourds } \\ \text { (métaux, métaux_précieux) } & \text { métaux-précieux } \\ \text { (métaux, métaux_lourds) } & \text { nickel } \\ \text { (métaux, métaux_précieux) } & \text { or } \\ \text { (métaux, métaux_précieux) } & \text { platine } \\ \text { (métaux, métaux_lourds) } & \text { zinc }\end{array}$

La mention de deux niveaux hiérarchiques est nécessaire à cause de la difficulté de manipulation des relations dans SATO.

\section{Une propriété Associés}

L'ajout d'une propriété Associés est possible, mais le nombre de valeurs associées à chaque forme risque d'être encombrant:

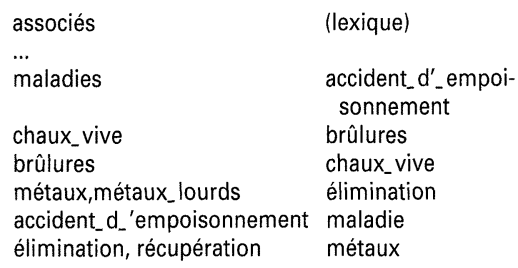

\section{Bilan sur les relations dans SATO}

La recherche d'information peut se faire soit sur chaque forme répertoriée dans le lexique de la base de données, soit sur sa propriété, de cette façon: tous les passages de textes contenant des occurrences ayant pour valeur de la propriété générique métaux précieux. Avec l'exemple fourni plus haut, on récupérerait toutes les concordances comportant argent, or, platine, métaux-précieux. Si I'on formulait la requête avec métaux, on obtiendrait tous les passages comportant un des termes du lexique affiché plus haut: argent, cadmium,chrome, etc.

L'ennui, c'est que SATO n'a pas été prévu, au départ, pour gérer ce genre de relations. II n'assure pas la réciprocité, ce qui expose le concepteur du thésaurus à toutes sortes d'erreurs, d'omissions, de contradictions.

L'apport réel du logiciel réside clairement dans le dépistage des relations, ce que ne font pas en général les autres logiciels. En somme, SATO et les logiciels documentaires sont 
complémentaires. Le premier assiste les opérations intellectuelles d'appréhension du contenu, de sélection des termes et de leurs relations, les seconds gèrent les résultats de ce travail, contrôlent les doublons, s'assurent de la réciprocité des relations, bref prennent en charge toutes les tâches, très lourdes si elles sont effectuées à la main, de vérification et de contrôle (Rohou, 1987). C'est ce qui a incité les responsables du projet VIXIT au Conseil du Trésor du gouvernement du Québec à coupler SATO et Seconde (de la firme Destin Inc.) pour l'analyse des textes en gestion des ressources humaines à l'aide $d^{\prime} u n$ vocabulaire contrôlé a posteriorí.

\section{Mise à jour : extraction des mots nouveaux}

La mise à jour du vocabulaire est facilitée dans SATO car, au fur et à mesure que des textes sont inclus dans le corpus, le logiciel permet de connaître les nouvelles formes introduites par ces textes et d'appliquer systématiquement les traitements seulement à ces formes. II suffit pour cela de faire deux sous-ensembles du corpus: le premier comporte tous les textes entrés avant la mise à jour, le second tous ceux qui ont été rajoutés. Lorsqu'on fait éditer le lexique, les formes qui ont une fréquence zéro dans le corpus le plus ancien correspondent aux formes nouvelles.

sous_ensemble1 sous_ensemble2 (nouvelles formes)

$\begin{array}{ccl}\text { fréq1 } & \text { fréq2 } & \\ 0 & 1 & \text { accumulateurs } \\ 0 & 1 & \text { actifs } \\ 0 & 1 & \text { admissible } \\ 0 & 1 & \text { agent_chelatant } \\ 0 & 1 & \text { agent_réducteur } \\ 0 & 1 & \text { aluminium } \\ 0 & 1 & \text { amalgame } \\ 0 & 1 & \text { anodique } \\ 0 & 1 & \text { anodisation } \\ 0 & 1 & \text { bioxyde_de_sodium } \\ 0 & 1 & \text { bisulfite_de_sodium } \\ 0 & 1 & \text { boue_contaminée } \\ 0 & 1 & \text { cadmium_résiduel }\end{array}$

\section{Les autres sources pour l'élaboration de thésaurus}

Les questions des utilisateurs

Nous n'avons parlé jusqu'ici que de l'exploitation des textes qui constituent la base de données. Cependant, la même méthodologie peut être appliquée aux questions des utilisateurs. Si on choisit le mode témoin au début d'une session de travail avec SATO, toutes les commandes et les réponses sont enregistrées. On peut donc trier et éditer le tout pour enrichir le thésaurus avec les termes - et éventuellement les relations. employés par les utilisateurs.

\section{Les sources terminologiques et lexicographiques}

Les banques de terminologie et les dictionnaires sur support lisible par ordinateur peuvent être exploités par SATO de la même façon que les textes. Leur régularité et leur normalisation sont d'ailleurs plus grandes. L'expérience rapportée dans ce même numéro par Serge Houde témoigne de l'intérêt de la démarche. Le fait que, dans la version du Robert- $E$ destinée au Macintosh, chaque mot clé de I'article soit directement accessible, accroît encore la facilité d'exploitation avec SATO. Un autre essai a été tenté avec la base de terminologie Termium sur CD-ROM ${ }^{7}$.

\section{Vers des thésaurus personnalisés}

On souligne de plus en plus le caractère " privé " des structures cognitives. Un terme peut, en effet, appartenir à plusieurs catégories, selon le point de vue adopté: " [...] different persons, in different occupations may possess different world views and make different demands upon sources of knowledge as a consequence ${ }^{8}$.

Contrairement à ce qu'affirme la norme ISO 2788 (Organisation internationale de normalisation, 1986), il n'existe pas à proprement parler de catégories a priori, mais des catégories imposées par des agents cognitifs dans un domaine donné, les auteurs des textes et reconnues par d'autres agents cognitifs, les lecteurs. Le choix des caractéristiques sur lesquelles s'appuie la catégorisation dépend du contexte d'utilisation (Frohmann, 1983). L'idéal pour un utilisateur est de pouvoir accéder au regroupement le plus parlant en fonction de la tâche à accomplir.

La technologie de I'hypertexte permet d'ores et déjà d'envisager une superposition de plusieurs visions différentes d'un domaine, comme celle d'un groupe d'utilisateurs par rapport à un autre (Agosti et al., 1989) ou bien d'un individu par rapport à un groupe (Belkin et al., 1991). Avec SATO, il est possible de catégoriser le vocabulaire de différentes façons et de conserver chacune des "prises de vue " sous forme de dictionnaire.

\section{En guise de conclusion partielle}

Lorsqu'il s'agit de construire un thésaurus de qualité, on ne peut pas compter sur la machine uniquement. On est confronté à ce défi qui, d'après Locke $^{9}$ accompagne tout effort de repérage en plein texte: "[...] the knowledge engineering that goes into constructing a first-class thesaurus of relevant concepts ". Mais tout comme les cogniticiens chargés d'élaborer des bases de connaissance, les spécialistes de l'information peuvent désormais s'appuyer sur des outils qui assistent l'appréhension du contenu des sources dépouillées pour structurer à la fois le lexique et les concepts d'un domaine, donc qui agissent en amont de l'analyse plutôt qu'en aval comme les logiciels documentaires traditionnels.

II ne reste pas moins beaucoup de recherches à effectuer sur les bases théoriques des thésaurus, sur leur contenu et l'organisation de leur contenu pour qu'ils puissent répondre adéquatement aux nouveaux besoins d'analyse de textes par ordinateur et de manipulation automatique pour la recherche documentaire:

[...] it may now be possible to leave to a human specialist's intervention only the more tricky cases, - interventions like disambiguation, word selection, etc., which then would have to be done by interaction.

So, the question being posed today is this: How must a machineoperated thesaurus look like, and can it be built and maintained, if it is to meet the needs of such machine

6. Le texte de Maurice Gingras dans cette livraison rend compte de cet important projet.

7. Hélène Tardif, Recherche en analyse documentaire; IConstitution d'une liste de termes de domaine à partir d'un corpus de la banque de terminologie Termium], [Montréal], Université de Montréal, École de bibliothéconomie et des sciences de l'information, avril 1991, 58 p. + annexes.

8. T.D. Wilson, "The Cognitive Approach to Information Seeking Behaviour and Information Use", Social Science Information Studies, vol. 4 (1984), 200

9. Christopher Locke, "The Dark Side... ", 200. 
or machine-aided natural language processing - among others: IR. [information retrieval] ${ }^{10}$.

\section{Aide à I'indexation}

\section{Le déclin de I'indexation humaine}

L'indexation humaine est devenue un véritable goulot d'étranglement. Beaucoup trop subjective, beaucoup trop Iongue, beaucoup trop coûteuse, elle est de plus en plus remplacée par l'indexation automatique rendue possible par la numérisation des données textuelles. Nous ne reprendrons pas ici la démonstration de l'impossibilité d'obtenir des résultats de qualité avec des méthodes aussi crues que l'indexation des chaînes de caractères autres que les formes fonctionnelles ou même de méthodes statistiques uniformes pour des types de discours aussi dissemblables que le droit, la littérature, la chimie, etc. et des genres aussi divers que l'article scientifique, la correspondance et le règlement administratif. Des processus cognitifs aussi complexes que la lecture et la condensation pour d'autres individus ne peuvent être formalisés, entre autres parce qu'ils sont encore partiellement inconnus et beaucoup trop complexes. D'autre part, les méthodes d'analyse linguistique des textes en langue naturelle sont encore très rudimentaires, malgré de belles réalisations. Nous avons montré par ailleurs que la meilleure solution résidait sans doute dans l'indexation assistée (Bertrand-Gastaldy, 1990b). De plus en plus de voix s'élèvent pour proposer une complémentarité des traitements linguistiques, statistiques et procéduraux, de type système expert (Chaumier et Dejean, 1992; Doszkocs, 1986; Meunier et al. , 1986, entre autres).

\section{L'indexation assistée par SATO}

Actuellement, avec SATO on peut surtout bénéficier d'une indexation qui recourt aux équivalents. C'est au concepteur du système d'indexation de déterminer la spécificité de son indexation en choisissant la granularité de son contrôle de vocabulaire. II peut considérer comme équivalents seulement les variantes flexionnelles, y ajouter certaines variantes dérivationnelles (pour regrouper par exemple les noms et les verbes d'action), les synonymes, les quasi-synonymes et même les antonymes, selon le taux de rappel souhaité, puisque la précision est assurée, en principe, par les formulations en langage naturel. La profondeur de l'indexation dépend en grande partie de la segmentation préalable des textes: on peut prendre comme unité documentaire le texte dans son entier, chaque chapitre, chaque paragraphe.

SATO va souligner dans les textes (seulement dans les titres et les soustitres si on désire une indexation superficielle) les formes répertoriées comme termes du domaine ou équivalents, ajouter la valeur de propriété à côté de la forme détectée et assigner en début de texte une forme unique, celle qui aura été déterminée comme le "descripteur" accepté. Voici un exemple de sous-titre ainsi indexé.

*index = revêtement_de_surface

*divis = stitre 1.2 Électrodéposition*index = revêtement_ de_surface

* divis = texte Le revêtement_de_surface est désigné par les termes d'électrodéposition, de galvanotechnique ou de galvanostégie. On peut le définir comme [...]

Il est possible de se fixer une grille d'indexation et de faire repérer systématiquement dans les textes les descripteurs qui correspondent aux "facettes" souhaitées, par exemple dans les conventions collectives: mesure, personnel concerné, personnel exclu, durée. Le rôle de l'indexeur se borne à réviser l'indexation en contexte, à éliminer les termes repérés qui ne correspondent pas au sujet, à rajouter des termes pour les concepts implicites ou ceux qui sont exprimés par des pronoms, à remplacer un terme général par un terme spécifique plus adéquat. Sa tâche est facilitée par le soulignement de toutes les occurrences qui ont déclenché l'indexation et par une fonction de "catégorisation" en contexte. Nous avons ainsi construit un petit modèle d'analyse de la correspondance de la division des évaluations environnementales au ministère de l'Environnement qui retient la phase du projet, s'il y a lieu, I'action (I'intervention sur l'environnement), l'objet de l'action, le lieu, les limites spatiales. L'exemple cidessous ilustre l'indexation d'une lettre après correction humaine (on voit toutes les annotations surimposées par les opérations d'attribution de valeurs de propriétés; l'indexat - ou ensemble de mots clés assignés - a été disposé automatiquement à la tête du texte et il est suivi du nom des facettes correspondantes):
${ }^{*}$ page $=$ let $2 / 1 / 4$

Sainte-Foy, le

*index = (réaménagement, route_148, Aylmer, chemin _Rivermead_et_Pont_Champlain $)^{*}$ facet $=($ action, objet, lieu, limites)

Destinataire 2

Aylmer*index $=$ Aylmer*facet $=$ lieu (Québec) $\overline{\mathrm{J} 9 \mathrm{H}-3 \mathrm{M} 2}$

Mxxxxx,

Le projet de réaménagement ${ }^{*}$ index $=$ réaménagement ${ }^{*}$ facet=action de la route $148 *$ index $=$ route $148 *$ facet $=$ objet entre le chemin Rivermead et le Pont Champlain *index = entre_le_chemin_Rivermead_et_le Pont Champlain*facet=limites à Aylmer*index $=$ Aylmer* facet $=$ lieu du ministère des Transports, est un projet assujetti à la procédure $d^{\prime}$ évaluation et d' examen des impacts sur $\mathrm{I}^{\prime}$ environnement ${ }^{*}$ index $=$ nil ${ }^{*}$ facet $=$ nil. [...]

Le ministère de I' Environnement est actuellement en attente de l'étude $d^{\prime}$ impact ${ }^{*}$ index $=$ nil ${ }^{*}$ facet $=$ nil. La procédure $d^{\prime}$ évaluation et $d^{\prime}$ examen des impacts sur I 'environnement ${ }^{*}$ index $=$ procédure_d'_évaluation_et_d'_examen_des_impacts_sur_l'_environnement * facet $=$ phase se poursuivra lorsque l'étude $d^{\prime}$ impact ${ }^{*}$ index $=$ nil*facet $=$ nil sera déposée au ministère de l'Environnement.

Veuillez agréer, Mxxxxx, l'expression de mes sentiments les meilleurs

\section{Auteur $Y$}

Lors de l'interrogation, selon le taux de rappel et de précision souhaités, on pourra interroger soit sur n'importe quel mot du texte, soit sur les descripteurs seulement (si on veut se restreindre à ce qui est vraiment thématique), soit sur l'ensemble des mots du texte et des descripteurs. On peut aussi demander la recherche sur tous les équivalents du descripteur. Les stratégies d'indexation ou "patrons de fouille" se présenteront ainsi :

1) concordance libre étude_d'_impact [récupère toutes les occurrences, telles quelles]

2) concordance libre étude $\$ d_{-} d_{-}$impact $\$$ [récupère toutes les occurrences, au singulier et au pluriel]

3) concordance libre $\$$ *équivalent=étude_ $d^{\prime}$ _impact [récupère toutes les occurrences équivalentes au descripteur, qu'elles représentent ou non le sujet du document (il n'y a pas eu d'indexation)]

4) concordance libre $\${ }^{*}$ index=étude_d'_impact [récupère toutes les formes équivalentes au descripteur assigné au texte ou au segment de texte, parce qu'il représentait le sujet du document selon l'indexeur qui a révisé les suggestions d'indexation de SATO]

Dans le cas de l'exemple ci-dessus, la lettre sera signalée par la stratégie no 3 (ou les stratégies 1 et 2):

\$*équivalent=étude_d'_impact

mais ne le sera pas si l'on précise qu'on ne cherche que les lettres dont le sujet principal porte sur cette phase du projet (stratégie no 4).

10. Winfred Schmitz - Esser, "Thesauri Facing... ", 130. 
Dans un logiciel documentaire comme Seconde, un menu présentera ces options et le logiciel ira chercher dans les champs indexation et/ou texte selon les spécifications de l'utilisateur. II pourra aussi, si on le demande, aller fouiller dans le thésaurus et afficher les descripteurs environnants. Comme le logiciel a une interface à menu, la plupart des utilisateurs le trouveront plus facile à utiliser; par contre, les experts seront privés de plusieurs fonctionnalités disponibles dans SATO. Dans I'application VIXIT, on recourt donc au logiciel SATO pour procéder à l'indexation, puis on transfère les résultats dans Seconde pour son exploitation.

\section{Indexation personnalisée}

L'indexation est ainsi accélérée et rendue plus uniforme. C'est une façon de procéder qui convient bien dans les contextes les plus familiers aux bibliothécaires: ceux où il faut desservir une clientèle assez nombreuse, qui interroge fréquemment et dont on peut bien cerner les besoins. Mais les textes informatisés dans les bureaux peuvent servir à toutes sortes de fins. Certains individus ont des préoccupations atypiques par rapport à leurs collègues. C'est ainsi que des étudiants de I'EBSI (École de bibliothéconomie et des sciences de l'information) ont dû proposer, pour une personne chargée de planification et d'études prospectives, un système qui repérait toutes les phrases susceptibles d'exprimer dans tout type de texte (produit à l'intérieur de l'organisation comme à l'extérieur) une notion de futur, de prévision. La solution a consisté à élaborer un dictionnaire pour les différentes formes susceptibles d'exprimer le temps et à mettre au point une série de stratégies dépistant la présence simultanée d'un de ces mots avec les terminaisons du futur ou du conditionnel (Chouinard, 1990; Domecq, 1989). La stratégie visant ce dépistage consiste donc en un mélange $d$ 'indexation au repérage et de catégorisation préalable du vocabulaire. Voici un exemple de phrases repérées:

II peut encore en être ainsi demain, dans la mesure où seront domestiquées les énergies solaire, géothermique, nucléaire...

Dans ces conditions, presque tous les systèmes seraient acceptables, si I'on y consacrait des ressources suffisantes.
Mais ce que nous indique l'analyse éco-énergétique, et qui échappait à tout autre indicateur, c'est que ce processus ne pourra pas se poursuivre indéfiniment.

On s'approche un tant soit peu de la situation idéale où l'indexation fournirait autant de données, accessibles d'autant de façons que le requerraient les différents problèmes à résoudre au sein de l'organisation. Habituellement, les gestionnaires de systèmes d'information en ont une image monolithique et cultivent une approche centralisatrice. Un bon système de gestion des ressources d'information devrait normaliser les pratiques d'analyse sans nier les différences d'approche nécessaires à I'efficacité: "You must allow for departmental and even individual requirements while still providing the standard basis for indexing " 11 .

Il faudra encore beaucoup d'études sur la façon de lire un texte, d'utiliser l'information, de poser des questions pour découvrir la diversité des approches. En rendant le texte accessible aussi facilement, on fait tomber le carcan dans lequel les systèmes bibliographiques enfermaient les lecteurs pour des raisons évidentes de limites technologiques et économiques. Avec des "boîtes à outils" du type de SATO, les utilisateurs atypiques peuvent mettre au point des analyseurs "sur mesure" qui se superposent aux analyseurs "prêts-àporter" pour la majorité (BertrandGastaldy, 1990b).

\section{Constitution d'index}

Un index peut être constitué qui comporte les références très précises (document, page, ligne et position dans la ligne) et peut être suivi d'un formatage avec un logiciel de traitement de texte:

\section{Concordance libre information politique}

information_politique

$\# 1 *$ page $=$ mediad $1 / 1 / 9 / 3$

\# 2 * page $=$ mediad $1 / 1 / 38 / 4$

\# $3 *$ page $=$ mediad $1 / 6 / 15 / 3$

$\# 4$ *page $=$ mediad $11 / 6 / 38 / 8$

$\# 5 *$ page $=$ mediad $2 / 8 / 34 / 2$

\section{Autres applications en analyse}

D'autres fonctionnalités de SATO peuvent être mises à profit: dans un service d'indexation, il peut être utile de disposer d'un diagnostic de lisibilité des textes pour répartir le travail en fonction de l'expérience ou des habiletés de chacun. II n'est pas non plus inintéressant de vérifier la lisibilité des résumés rédigés par le service.

Comme certains documents administratifs connaissent de nombreuses versions, le marquage par un souligné des ajouts dans une version par rapport à une autre aide à évaluer rapidement la nécessité de réindexer la nouvelle version.

Nous utilisons actuellement SATO et SPSS ${ }^{12}$ pour analyser les résultats des analyses effectuées par des indexeurs afin de détecter les corrélations entre une rubrique de classification et les mots du texte, plus précisément, les mots dans certaines subdivisions de texte. De la sorte, il sera possible de concevoir une série de règles qui déclencheront l'attribution automatique de telle ou telle rubrique sur la base de la coprésence de certaines des unités lexicales discriminantes. En outre, le système expert pourra exploiter des relations thésaurales autres que les relations d'équivalence pour l'indexation. La présence simultanée d'un certain nombre de termes spécifiques pourra conduire à l'assignation du générique. La coprésence de tel ou tel terme dans un contexte déterminé pourra contribuer à désambiguïser un polysème.

\section{Le repérage}

Nous avons montré comment on peut recourir à SATO pour mener à bien les principales activités documentaires: l'élaboration de thésaurus, l'indexation et, au travers de ces deux fonctions, nous avons illustré certaines des possibilités d'exploration des corpus qui constituent autant de stratégies de recherche. Le lecteur se sera sans doute rendu compte de la multiplicité des combinaisons possibles. Nous allons récapituler les caractéristiques les plus saillantes.

11. Michael O'Shea, "Simply Defined: Nonsense Filing Everyone Can Live With", Office Equipment \& Methods, (November 1989), 18.

12. II s'agit d'un projet intitulé "Conception d'un système expert d'aide à l'analyse (tri, classification et indexation) des jugements, subventionné par le CEFRIO, la SOQUIJ (Société québecoise d'information juridique) et le ministère des Communications du Québec. II est dirigé par S. Bertrand-Gastaldy et JeanGuy Meunier et inclut la participation de François Daoust, Luc Dupuy, Yves Khawam, Gracia Pagola et Louis-Claude Paquin. 
On peut visualiser les mots dans le texte ou dans le lexique trié par ordre alphabétique, de fréquence, de longueur et d'après les propriétés assignées.

Plusieurs mots peuvent être inclus dans la stratégie de recherche avec des conditions sur leur position respective: dans n'importe quel ordre (concordance libre), dans l'ordre de la déclaration (concordance ordonnée), dans une position d'adjacence (concordance stricte).

La fouille sur les chaînes de caractères bénéficie de la possibilité de troncature à droite et à gauche, comme nous l'avons vu plus haut, ainsi que du masque:

\section{La commande:}

\section{Écrire lexique pruden-e \\ peut récupérer: \\ prudence \\ prudente}

Si I'on cherche toutes les formes de 3 lettres, on demandera:

\section{Écrire lexique \$--}

ce qui donnera:

$\begin{array}{rl}\text { fréq } & \\ 7 & \text { aux } \\ 1 & \text { bon } \\ 1 & \text { cas } \\ 7 & \text { ces } \\ 143 & \text { des } \\ 2 & 100\end{array}$

Avec la commande suivante où :

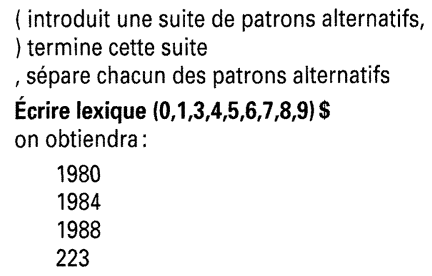

I sert à inclure dans la stratégie un caractère spécial (comme un signe de ponctuation) ou une majuscule. La commande:

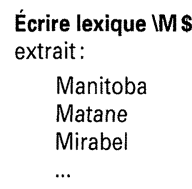

Lorsqu'on cherche une valeur de propriété symbolique (qui porte un nom), on peut utiliser $=$ ( pour égal) et $\sim$ (pour différent). Sil'on veut toutes les formes qui sont des noms, on écrit:

$S^{*}$ gramr $=$ nomc

et on aboutit à ce genre de résultat:

$\begin{array}{cll}\text { fréq } & \text { gramr } & \\ 1 & \text { nomc } & \text { administrateur } \\ 2 & \text { nomc } & \text { administrateurs } \\ 2 & \text { nomc } & \text { agence } \\ 1 & \text { nomc } & \text { application }\end{array}$

Les opérateurs disponibles pour les propriétés numériques sont:

$\begin{array}{ll}= & \text { pour: égal } \\ < & \text { pour: } \text { plus petit que } \\ > & \text { pour: plus grand que } \\ \text {, pour l'opérateur logique ou } & \\ \text { \$*fréquence>5 } & \\ \text { fréq } & \\ 7 & \text { accès } \\ 10 & \text { acteurs } \\ 11 & \text { agences } \\ 28 & \text { analyse }\end{array}$

Nous avons dit plus haut que I'on peut associer une fouille sur les caractères et une fouille sur les propriétés. Voici un exemple où I'on cherche tous les mots qui ont reçu la valeur infinitif et qui se terminent par ir, er, oir, re:

l(ir,er,oir,re) * gramr=infinitif

$\begin{array}{cll}\text { fréq } & \text { gramr } & \\ 1 & \text { infinitif } & \text { avoir } \\ 1 & \text { infinitif } & \text { conclure } \\ 1 & \text { infinitif } & \text { contenir } \\ 2 & \text { infinitif } & \text { penser }\end{array}$

Cet autre exemple combine une troncature à gauche sur une chaîne de caractères, une valeur de propriété grammaticale et une fréquence:

Écriie lexique lation* gramr $=$ nomc $*$ fréqtot $>5$

$\begin{array}{rll}\text { fréq } & \text { gramr } & \\ 13 & \text { nomc } & \text { communication } \\ 101 & \text { nomc } & \text { information } \\ 10 & \text { nomc } & \text { présentation } \\ 6 & \text { nomc } & \text { relation } \\ 6 & \text { nomc } & \text { situation }\end{array}$

Dans le texte, on peut délimiter le contexte dans lequel s'effectuera la fouille: contexte numérique en fonction d'un nombre fixé par l'utilisateur de mots avant et après le mot cherché ; contexte délimité en fonction de la présence dans le texte de caractères délimiteurs (comme les signes de ponctuation forte ou faible), ce qui permet par exemple de chercher dans des phrases, des portions de phrases ou des paragraphes; contexte homogène obtenu en fonction des valeurs d'une propriété textuelle donnée: les textes, les chapitres, les notices, les e ées de dictionnaire, etc. On peut aiıcher ou imprimer également toute portion de texte qui correspond à une valeur de propriété textuelle, par exemple tous les titres:

\section{Écrire texte $\$ *$ zone $=\mathrm{tm}$}

${ }^{*}$ notice $=1 *$ zone $=\mathrm{tm}$ Analyse du traitement journalistique de l'information politique au Québec : le cas du projet de restructuration scolaire de 1982

* notice $=2^{*}$ zone $=\mathrm{tm}$ La position canadienne face au nouvel ordre mondial de l'information et de la communication

*notice $=3^{*}$ zone $=\mathrm{tm}$ L'information administrative et les moyens de puissance

On peut ensuite extraire les formes lexicales qui proviennent des titres:

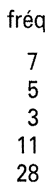

$\begin{array}{ll}\text { zone } & \\ \mathrm{tm} & \text { accès } \\ \mathrm{tm} & \text { activités } \\ \mathrm{tm} & \text { administrative } \\ \mathrm{tm} & \text { agences } \\ \mathrm{tm} & \text { analyse }\end{array}$

On n'en finirait pas de donner des exemples de combinaisons diverses. II faut laisser aller son imagination, ce qui n'est pas aussi aisé lorsqu'on est conditionné par les contraintes des logiciels documentaires traditionnels !

La souplesse d'exploration de SATO peut d'ailleurs se transformer en difficulté d'utilisation, car il faut maîtriser une syntaxe plus riche (tant au niveau des unités que des opérateurs pour les manipuler) que celle qu'offrent d'habitude les logiciels documentaires. C'est pourquoi SATO est bien adapté pour les concepteurs, les chercheurs et fouineurs de tout acabit! Pour les autres, ceux qui ont d'autres occupations, il est possible de dissimuler la plupart des démarches sous des macro-commandes qui rendent le logiciel transparent, ou bien de déverser les résultats d'analyse dans un logiciel documentaire qui offre, par contre, beaucoup moins de diversité d'approches.

\section{Au-delà des procédures d'analyse du contenu: les questions théoriques}

Au-delà des procédures pour faciliter l'analyse du contenu, le dépouillement automatique de textes intégraux soulève des questions théoriques importantes que nous mentionnons brièvement ( nous les avons développées davantage dans BertrandGastaldy et Pagola, 1992).

En ce qui concerne le contenu d'un thésaurus, on peut s'interroger, par exemple sur le type d'unités lexicales à conserver, sur l'inclusion des verbes, des adjectifs et des adverbes à côté des noms pour une meilleure caractérisation d'un domaine. On peut aussi se demander comment interrelier les 
termes propres au domaine, le vocabulaire de la langue commune et les mots sélectionnés de façon privilégiée pour accompagner les termes du domaine (ce que les terminologues appellent les co-occurrents). La question de la finesse des étiquettes de relations en fonction des conditions d'utilisation, notamment dans les systèmes à base d'inférence, est également débattue. Les liens entre thésaurus, terminologies et bases de connaissances font également I'objet de recherches.

Pour ce qui est des sources à exploiter en vue de colliger les termes du thésaurus, on a mentionné les textes de la base de données à interroger, les dictionnaires et banques de terminologie, les questions des utilisateurs. On se doute qu'on ne peut manipuler au hasard les sources de données. II faut se poser des questions sur leur validité à alimenter un thésaurus et, plus fondamentalement sur la fonction des thésaurus, donc sur le genre de connaissances à $y$ inclure. Les relations qu'ils mettent en évidence sontelles des relations communément acceptées dans la vie quotidienne, dans le domaine de spécialité, dans la base de données particulière à laquelle on veut faciliter l'accès? Ces relations concernent-elles les concepts qui seraient exprimés par les termes ou les relations entres les différentes unités lexicales, ou encore les deux? Rien de tout cela n'est clarifié dans la littérature sur les thésaurus.

Les phénomènes textuels qu'il faudrait maîtriser pour arriver à une indexation automatique capable d'extraire avec précision le sujet du document ne sont pas abordés depuis très longtemps dans la littérature en sciences de l'information. Mais on sait mieux désormais comment procèdent les indexeurs humains. Avec toutes leurs connaissances de la langue, du domaine, des conditions de production des textes et du contexte d'utilisation, ils réussissent en principe à reconnaître les équivalences sémantiques entre un terme et sa définition, les périphrases et les paraphrases, à distinguer les nuances fines entre énoncés presque semblables, à détecter les concepts implicites, à suppléer aux ellipses, à rattacher correctement les pronoms à leurs référents, à laisser de côté les informations superflues ou redondantes et à intégrer progressivement les détails présentés dans les micro-propositions pour dégager le sens général, les macro-propositions. Ils filtrent l'information la plus pertinente pour les utilisateurs dont ils connaissent bien les besoins. Le problème se pose donc de savoir jusqu'où et comment on peut le mieux les assister dans ces tâches complexes, tout en sachant qu'on ne peut guère les remplacer entièrement, sauf dans des cas où les sous-titres fournissent une sorte d'auto-indexation ou encore lorsqu'une indexation de piètre qualité n'est pas trop pénalisante. La solution à privilégier doit s'appuyer sur une analyse coûts-bénéfices et dépend de la disponibilité de logiciels évolués.

\section{Conclusion}

SATO a servi à illustrer le parti que I'on peut tirer d'outils d'aide à l'analyse de contenu pour mener à bien certaines tâches reliées à l'analyse et au repérage.

Le logiciel n'est pas sans défaut. Le vocabulaire des commandes peut rebuter le spécialiste de l'information documentaire. Une grosse amélioration de l'interface serait nécessaire. II faudrait lui ajouter d'autres modules, en particulier un analyseur morphosyntaxique. Des systèmes comme ALETH de la firme ERLI, SPIRIT, FASIT ou INDEX-D procèdent à des analyses plus fines des lexies complexes.

Par contre, il offre une panoplie étonnante d'outils destinés à des approches multiples des textes. De plus, il peut être couplé à des logiciels aussi bien en amont (éditeurs comme PE, manipulateurs de chaînes de caractères comme ICON) qu'en aval (logiciel statistique comme SPSS, logiciel documentaire comme Seconde, coquille de système expert, etc.), ce qui va dans le sens d'une conception modulaire des instruments de traitement adaptée à la diversité des objectifs poursuivis. L'un des bénéfices que l'on peut tirer de son utilisation est d'ordre pédagogique. Nous prenons conscience de la complexité de l'objet textuel et du nombre de connaissances déclaratives et procédurales qu'il faut ajouter pour reproduire un tant soit peu certaines étapes de l'analyse humaine.

Avec de tels outils à notre disposition, nous nous rendons bien compte qu'à l'avenir ce n'est plus tant la mise en oeuvre de certains traitements qui posera problème mais plutôt les fondements théoriques de nos façons de fonctionner et de nos outils traditionnels. De beaux défis sont à I'horizon!

\section{BIBLIOGRAPHIE}

Agosti, M. et al., "New Prospects in Information Retrieval Techniques: a Hypertext Prototype in Environmental Law ", in Online Information 89, Proceedings of the 13th International Online Information Meeting, London, 12-14 December 1989 , p. $483-494$

Association française de normalisation. Règles d'établissement des thésaurus monolingues. Z 47-100, Paris, AFNOR, décembre 1981.

Auger, Pierre. "Terminographie et lexicographie assistées par ordinateur; état de la situation et prospectives". Actes du Colloque Les industries de la langue: Perspectives des années 1990, Montréal, 21-24 novembre 1990. [Québec], Gouvernement du Québec, p.659680.

Balcer, Madeleine et GONIN, Jean-Paul. "Réactions de l'usager face à l'utilisation du système de repérage en mode dialogué, BADADUO". Documentaliste, vol. 16, no 2 (mars-avril 1979), 55-61.

Belkin, N.J. "User Interfaces for Information Systems". Journal of Information Science vol. 17, (1991), 327-344.

Bertrand-Gastaldy, Suzanne, 1992. Le contrôle du vocabulaire et l'indexation assistés par ordinateur; une approche méthodologique pour I'utilisation de SATO. Avec la collaboration de Gracia Pagola. [Montréal], Université de Montréal, École de bibliothéconomie et des sciences de l'information, janvier 1992, pagination variée [612 p.] [en pré-édition; édition définitive : été 1992].

Bertrand-Gastaldy, Suzanne, 1984. "Les thésau rus de recherche; des outils pour l'interrogation en vocabulaire libre ". Argus, vol. 13, no 2 (1984), 51-58.

Bertrand-Gastaldy, S., 1990a "L'évolution de la gestion de l'information documentaire sous l'impulsion des nouvelles technologies". Terminogramme; Bulletin d'information terminologique et linguistique, vol. 55 (mars 1990), 25-31.

Bertrand-Gastaldy, S., 1990b. "L'indexation assistée par ordinateur: un moyen de satisfaire les besoins collectifs et individuels des utilisateurs de bases de données textuelles dans les organisations ". ICO Québec; Intelligence artificielle et sciences cognitives au Québec; 2 (3), septembre 1990, 71-91.

Bertrand-Gastaldy, S. et Pagola, G. 1992. "L'élaboration et la gestion d'un vocabulaire de domaine dans le contexte des bases de données textuelles: remises en question et méthodologies ". Colloque Repérage de l'infor mation textuelle organisé conjointement par I'Hydro-Québec et le ministère des Communications du Québec, Montréal, le 18 septembre 1991. [Montréal], Hydro-Québec, mars 1992, 51-71.

Blair, David, C., 1986. "Full text retrieval: evaluation and implications". International Classification, vol. 13, no. 1 (1986), 18-23. 
Blair, David, C. and Maron, M.E., 1985. "An evaluation of retrieval effectiveness for a fultext document retrieval system ". Communications of the ACM, vol. 28, no. 3 (March 1985), 289-299.

Bourque, G. et Duchastel, J.,1988. "Restons traditionnels et progressifs". Pour une nouvelle analyse du discours politique; le cas du régime Duplessis au Québec. Montréal, Boréal 1988. 399 p.

Chaumier, Jacques et Déjean, Martine, 1992. "L'indexation assistée par ordinateur: principes et méthodes ". Documentaliste; sciences de l'information, vol. 29, no 1 (1992), 3-6.

Chouinard, Daniel, 1990. La notion d'avenir en français ; une exploration au moyen du logicie SATO. [Montréal], Université de Montréal, École de bibliothéconomie et des sciences de l'information, avril 1990. $33 \mathrm{p}$.

Domecq, Marie-Cécile, 1989. SATO; exemple d'une application: détermination de la valeur "futur" dans des textes administratifs. [Montréal], Université de Montréal, École de bibliothéconomie et des sciences de l'information, décembre $1989.75 \mathrm{p}$.

Doszkocs, Tamas E., 1986. « Natural language processing in information retrieval ". Journal of the American Society for Information Science, vol. 37, no. 4 (1986), 191-196.

Duchastel, Jules, 1991. "Étude d'un corpus de dossiers de la cour juridique de Winnipeg à l'aide du système d'analyse de textes par ordinateur (SATO) ". Actes du Colloque Jornadas Internacionales de Análisis de Datos Textuales, Universitat Politécnica de Catalunya, Barcelona (Espagne), 1991.

Frohmann, Bernhard P., 1983. "An investigation of the semantic bases of some theoretical principles of classification proposed by Austin and the CRG ". Cataloging and Classification Quarterly, vol. 4, no. 1 (Fall 1983), 11-27.

Garson, L.R. and Love, R.A., 1985. "Full text searching of the ACS journals online: use and abouse ", in Online 1985 Conference Proceedings. New York, November 4-6, 1985, Weston, Conn., Online Inc., (1985), 116-119.

Lancaster, F.W., 1977. "Vocabulary control in information retrieval systems". Advances in Librarianship, vol. 7 (1977), 1-40.

Laroche, Léo, 1990. "Calibrage des textes et lisibilité ". ICO Québec; Intelligence artificielle et sciences cognitives au Québec; vol. 2, no 3 (septembre 1990), 114-118.

Meunier, J.-G. et al., 1987. "A call for enhanced representation of content as a means of improving on-line full-text retrieval ". International Classification, vol. 14, no. 1 (1987), 210.

Organisation internationale de normalisation, 1986. Principes directeurs pour l'établissement et le développement de thésaurus monolingues. ISO 2788 (F). [Genève], ISO, 1986. 32 p.
Paquin, Louis-Claude et al., 1990. "Analyse de texte et acquisition des connaissances: aspects méthodologiques ". ICO Québec; Intelligence artificielle et sciences cognitives au Québec, vol. 2, no 3 (septembre 1990), 95-113.

Ranjard, Sophie, 1991. "L'indexation manuelle: une valeur ajoutée". Archimag. Hors série, novembre 1991.

Richer, Pierre, 1986. "La création automatique d'un thésaurus de recherche". Argus, vol. 15 no 1 (1986), 13-19.

Rohou, Cécile, 1987. "La gestion automatisée des thésaurus; étude comparative de logiciels ". Documentaliste, vol. 24, no 3 (mai-juin 1987), 103-108.

Salton, Gerard, 1971. The SMART Retrieval System. Englewood Cliffs, NJ, Prentice-Hall, 1971.

Sparck-Jones, Karen, 1971. Automatic Keyword Classification for Information Retrieval. London, Archon Books, 1971

Tenopir, Carol, 1985. "Searching Harvard Business Review online; lessons in searching a full text database ". Online, vol. 9, no. 2 (1985), 71-78.

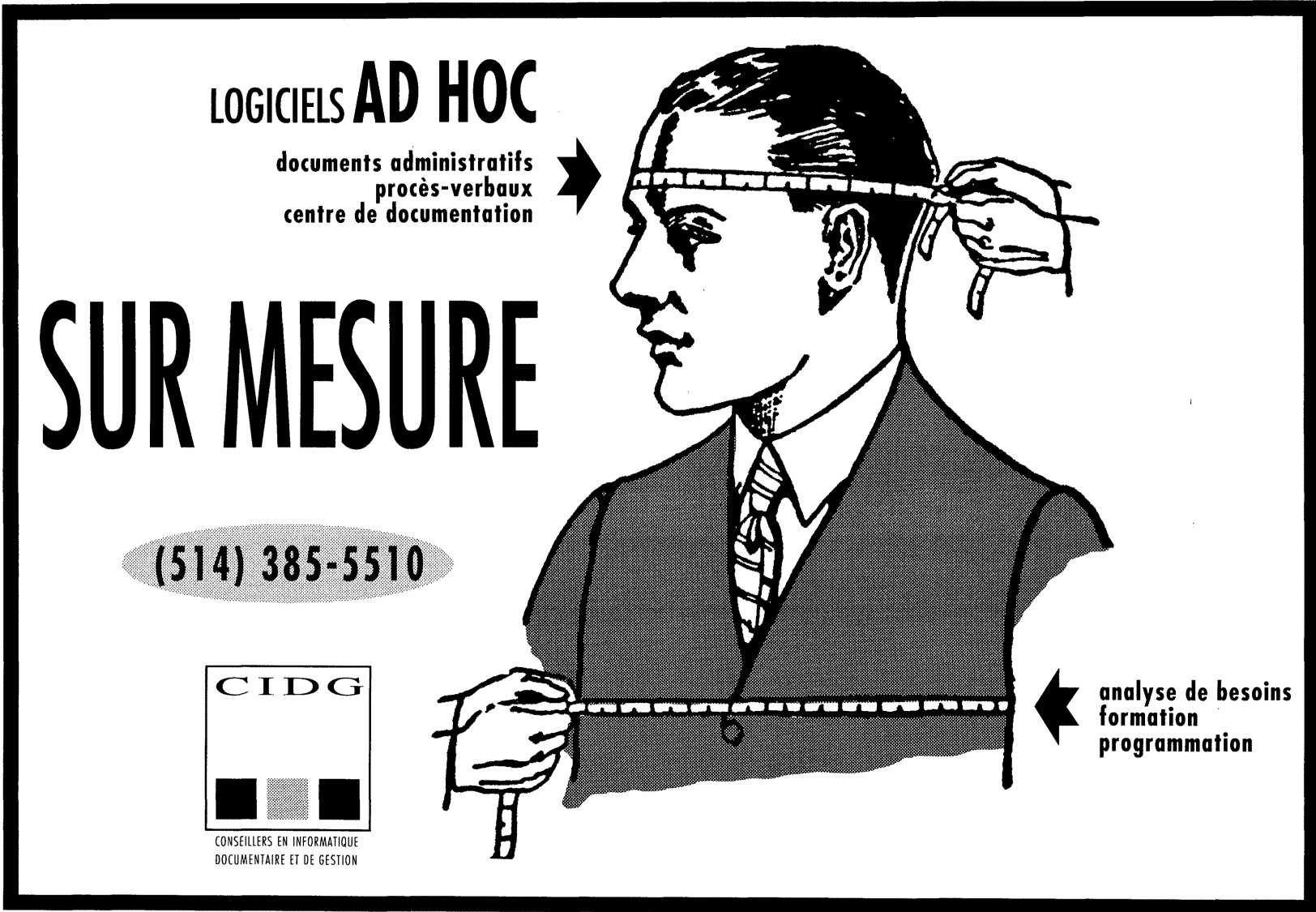

